\title{
Curcumin Nanoparticles Inhibiting Ferroptosis for the Enhanced Treatment of Intracerebral Hemorrhage
}

\author{
Cong Yang ${ }^{1, *}$ \\ Mengmeng $\mathrm{Han}^{1,2, *}$ \\ Ruoyu $\mathrm{Li}^{1, *}$ \\ Ligui Zhou ${ }^{1,3}$ \\ Ying Zhang ${ }^{1,2}$ \\ Lining Duan ${ }^{1,4}$ \\ Shiyu Su ${ }^{1,4}$ \\ Min $\mathrm{Li}^{1,4}$ \\ Qi Wang' \\ Tongkai Chen (D) ${ }^{1, *}$ \\ Yousheng Mo $\mathbb{D}^{5, *}$
}

'Science and Technology Innovation Center, Guangzhou University of Chinese Medicine, Guangzhou, 51 0405, People's Republic of China; ${ }^{2}$ The First Affiliated Hospital of Guangzhou University of Chinese Medicine, Guangzhou, 5I0405, People's Republic of China; ${ }^{3}$ Laboratory Animal Center, Guangzhou University of Chinese Medicine, Guangzhou, 510405, People's Republic of China; ${ }^{4}$ Clinical Medical College of Acupuncture Moxibustion and Rehabilitation, Guangzhou University of Chinese Medicine, Guangzhou, 5 10405 , People's Republic of China; ${ }^{5}$ The Second Affiliated Hospital of Guangzhou University of Chinese Medicine, Guangzhou, 510120 , People's Republic of China

*These authors contributed equally to this work
Background: Intracerebral hemorrhage (ICH) is a form of severe stroke, the pathology of which is tied closely to a recently discovered form of programmed cell death known as ferroptosis. Curcumin (Cur) is a common phenolic compound extracted from the rhizome of Curcuma longa capable of hematoma volume and associated neurological damage in the context of ICH. Despite exhibiting therapeutic promise, the efficacy of Cur is challenged by its poor water solubility, limited oral bioavailability and inability to efficiently transit across the physiological barriers. Polymer-based nanoparticles (NPs) have widely been employed to aid in drug delivery efforts owing to their ideal biocompatibility and their ability to improve the bioavailability and pharmacokinetics of specific drugs of interest.

Methods: In this study, we encapsulated Cur in NPs (Cur-NPs) and explored the effect of these Cur-NPs to enhance Cur delivery both in vitro and in vivo. Furthermore, we evaluated the anti-ferroptosis effect of Cur-NPs in ICH model mice and erastin-treated HT22 murine hippocampal cells.

Results: The resultant Cur-NPs were spherical and exhibited a particle size of $127.31 \pm 2.73$ $\mathrm{nm}$, a PDI of $0.21 \pm 0.01$ and a zeta potential of $-0.25 \pm 0.02 \mathrm{mV}$. When applied to Madin Darby canine kidney (MDCK) cells in vitro, these Cur-NPs were nonspecifically internalized via multiple endocytic pathways, with plasma membrane microcapsules and clathrinmediated uptake being the dominant mechanisms. Within cells, these NPs accumulated in lysosomes, endoplasmic reticulum and mitochondria. Cur-NPs were capable of passing through physiological barriers in a zebrafish model system. When administrated to C57BL/ 6 mice, they significantly improved Cur delivery to the brain. Most notably, when administered to ICH model mice, Cur-NPs achieved superior therapeutic outcomes relative to other treatments. In a final series of experiments, these Cur-NPs were shown to suppress erastininduced ferroptosis in HT22 murine hippocampal cells.

Conclusion: These Cur-NPs represent a promising means of improving Cur delivery to the brain and thereby better treating $\mathrm{ICH}$.

Keywords: Intracerebral hemorrhage, nanoparticles, curcumin, brain delivery, blood-brain barrier, ferroptosis

\section{Introduction}

Intracerebral hemorrhage $(\mathrm{ICH})$ is a severe form of stroke accounting for $15-25 \%$ of total stroke incidents, ${ }^{1}$ with affected patients exhibiting a 1-year survival rate of under $40 \%{ }^{2}$ Despite being a major cause of human morbidity and mortality, the mechanisms whereby ICH induces widespread neurological damage remain incompletely understood. During the acute phase of ICH immediately following the initial
Correspondence: Tongkai Chen; Yousheng Mo

Email chentongkai@gzucm.edu.cn; 378480308@qq.com 


\section{Graphical Abstract}

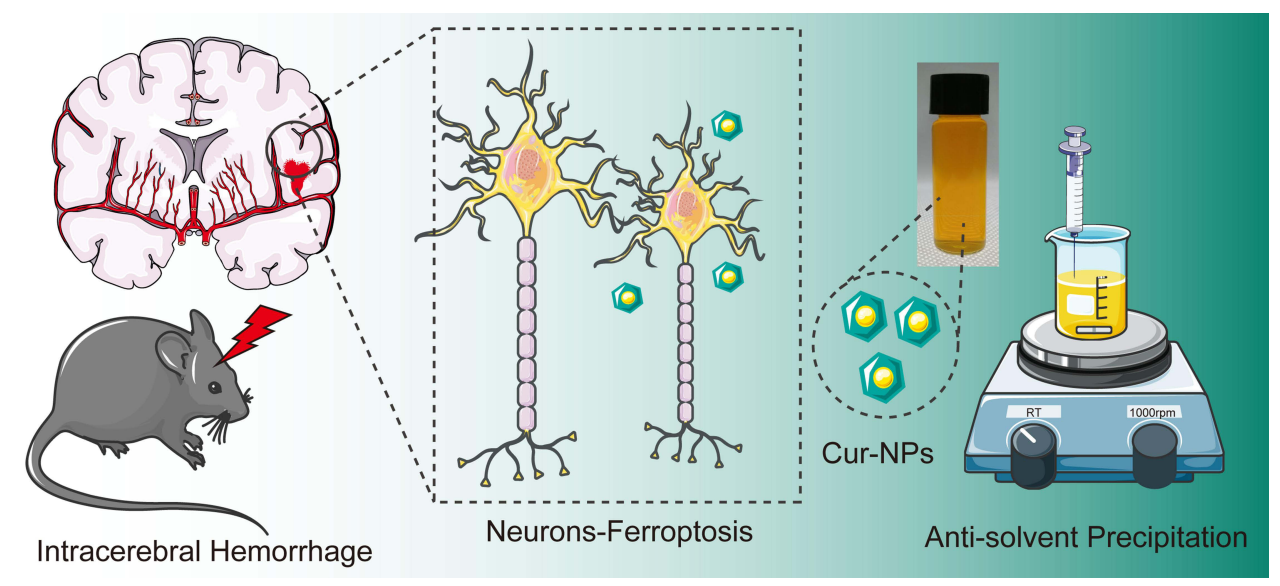

hemorrhage, hemoglobin undergoes rapid degradation which yields large volumes of iron within the extracellular space, together with biliverdin and carbon monoxide. ${ }^{3}$ There is evidence that this resultant iron overload can drive consequent edema in the perihematomal region, contributing to the accumulation of peroxide and widespread cellular death. ${ }^{4}$ Ferroptosis is a recently identified form of iron-dependent programmed cell death that can be induced in response to lipid peroxidation. ${ }^{5}$ Recent studies demonstrated that ferroptosis plays an integral role in the pathology of $\mathrm{ICH}^{6}{ }^{6}$ and targeting this cell death pathway may thus represent an effective means of treating this deadly form of stroke. ${ }^{7}$

Surgical hematoma evacuation can achieve some level of therapeutic efficacy in ICH patients, but this approach is very expensive and associated with substantial risk, limiting its utility for most patients. ${ }^{8-10}$ There is currently a lack of effective pharmacological treatments approved for the treatment of $\mathrm{ICH}^{11}$ and the development of drugs to treat this condition is hampered by limited oral bioavailability and the difficulties associated with delivering drugs across the blood-brain barrier (BBB). ${ }^{12,13}$ To overcome these challenges, many researchers have sought to derive novel pharmaceutical compounds from traditional herbal medicines. ${ }^{14}$

Curcumin (Cur) is a widespread phenolic compound that is extracted from the rhizome of Curcuma longa and that reportedly exhibits robust antioxidant, antiinflammatory, and neuroprotective activity when employed in pharmacological contexts. ${ }^{15-17}$ When employed to treat ICH model mice, Cur was able to effectively reduce the hematoma volume and associated neurological injury in these animals. ${ }^{18}$ However, the efficacy of Cur is profoundly hampered by its unwanted water solubility, poor oral bioavailability, and inability to efficiently transition across the BBB and other physiological barriers. ${ }^{19}$ Prior studies have sought to improve the therapeutic utility of Cur by encapsulating it within polymeric micelles, ${ }^{20}$ liposomes $^{21}$ and solid lipid nanoparticles. ${ }^{22}$ While these approaches were able to enhance Cur bioavailability to some degree, the associated Cur pharmacokinetic profiles and the ability of these particles to efficiently cross physiological barriers remain to be clarified. As such, there is a clear need for the development of new therapeutic approaches to improving the oral bioavailability and brain accumulation of Cur as a means of improving $\mathrm{ICH}$ patient outcomes.

Polymer-based nanoparticles (NPs) are highly biocompatible and are commonly used as tools to facilitate drug delivery, having received approval from the US Food and Drug Administration for medical use. ${ }^{23-26}$ NPs have previously been demonstrated to significantly enhance solubility, bioavailability, efficacy, and pharmacokinetic profiles of encapsulated drugs. ${ }^{27,28}$ These NPs are also highly multifunctional, less toxic, and less immunogenic as compared to many other drug delivery nanoplatforms. ${ }^{29}$ NPs can also be prepared by an inexpensive and easy-toperform antisolvent precipitation approach. ${ }^{30,31}$

Herein, we prepared Cur polymer-based NPs (CurNPs) via an antisolvent precipitation approach. We then utilized Madin Darby canine kidney (MDCK) cells as an 
in vitro model system to study drug absorption, as these cells exhibit tight junctions and a polarized mucus layer similar to intestinal epithelial cells. ${ }^{32}$ The endothelial BBB in zebrafish is similar to that observed in higher vertebrates, ${ }^{33}$ enabling us to use these animals as a model system to monitor Cur-NPs in vivo distribution profiles and elimination. We then explore the pharmacokinetics of Cur-NPs in the brain and plasma of treated mice, and evaluated the neuroprotective efficacy of these NPs in a murine ICH model system. The ability of these Cur-NPs to suppress ferroptosis was then assessed in vitro using HT22 murine mouse hippocampal cells.

\section{Materials and Methods Materials}

Cur and PVP-90 (Polyvinylpyrrolidone K90) were purchased from Nantong Feiyu Biological Technology Co., Ltd. (Nantong, China). MPEG-PTMC diblock copolymer, (Monomethoxy polyethylene glycol (MPEG), MW, 2000 Da; Poly (trimethylene carbonate) (PTMC), MW 16000 Da) was purchased from the Jinan Daigang Biomaterial Co., Ltd (Jinan, China). 5-(N-ethyl-N-isopropyl)-amiloride (EIPA), LY294002 (LY), Chlorpromazine (CPZ), Hypertonic sucrose (HS), MTT solution, and Methyl- $\beta$ cyclodextrin (M $\beta C D)$ were purchased from Sigma Aldrich (MO, USA). Coumarin 6 (C6), Lysotracker, ER tracker and Mitotracker were obtained from Molecular Probes Inc. (OR, USA). Erastin was purchased from MCE (NJ, USA). Live/Dead detection kit was obtained from KeyGene Biotech (Nanjing, China). Collagenase Type IV was purchased from ThermoFisher Scientific (NJ, USA). Nissl staining kit was purchased from Beyotime Biotechnology Co. Ltd. (Shanghai, China). Prussian blue staining kit was procured from Servicebio (Wuhan, China). H\&E staining kit was obtained from Leagene Biotechnology (Beijing, China). Primary antiGPX4 was obtained from Abcam, Inc. (Cambridge, UK). Secondary anti-rabbit IgG (Alexa Fluor 594) was purchased from Cell Signaling Technology (MA, USA). MDCK cells were purchased from the American Type Culture Collection (MD, USA). Mouse hippocampal neuronal cell line HT22 was purchased from the GuangZhou Jennio Biotech Co., Ltd (Guangzhou, China).

\section{Cur-NPs Preparation and Characterization}

An antisolvent precipitation approach was used to prepare Cur-NPs. Briefly, acetone was used to dissolve Cur
$(20 \mathrm{mg} / \mathrm{mL})$ and PEG-PTMC $(20 \mathrm{mg} / \mathrm{mL})$ as the organic phase, with water being used to dissolve PVP k90 (2 mg/ $\mathrm{mL}$ ) to prepare an aqueous phase. A total of $0.2 \mathrm{~mL}$ of the organic phase solution was then quickly injected into $10 \mathrm{~mL}$ aqueous phase solution, and the resultant mixture was stirred at $1000 \mathrm{rpm} / \mathrm{min}$ stirring to obtain the Cur-NPs. Additionally, C6 and mPEG-PTMC were mixed in the organic phase at a ratio of 1:30 to make C6-NPs. To remove the residual solvent, the obtained C6-NPs were stirred in the dark at room temperature for $2 \mathrm{~h}$. The physicochemical properties of Cur-NPs were analyzed, including the morphology by a transmission electron microscope (TEM), the particle size, PDI and zeta potential by dynamic laser scattering (DLS), and the crystalline patterns by powder X-ray diffraction (XRD).

\section{Evaluation of Cur-NPs Endocytic Uptake}

To assess the uptake of Cur-NPs in vitro, MDCK cells were treated for $30 \mathrm{~min}$ with five different inhibitors of endocytic pathways (EIPA, LY, CPZ, HS and M $\beta C D$ ). ${ }^{34}$ These cells were then treated for $1 \mathrm{~h}$ with C6-NPs, after which the supernatant was removed and cells were washed thrice with PBS. Cells were then fixed for 5 min with $4 \%$ paraformaldehyde (PFA) and imaged via confocal laser scanning microscopy (CLSM; TCS SPE II, Leica, Germany).

\section{Analysis of Cur-NPs Accumulation with Cells}

MDCK cells were plated onto coverslips in 24-well plates $\left(5 \times 10^{4}\right.$ cells/well $)$ and cultured in the presence of C6-NPs in complete medium at $0.5,1.0$ and $2.0 \mu \mathrm{g} / \mathrm{mL}$. After 10 , 30 or $60 \mathrm{~min}$, media was removed from these cells and they were fixed with $4 \%$ PFA prior to imaging at $488 \mathrm{~nm}$ via fluorescence microscopy (DMi8, Leica, Germany). The accumulation of these NPs within the lysosomes, ER and mitochondria were assessed by treating cells for 2 $\mathrm{h}$ with the Lysotracker, ER tracker and Mitotracker dyes. ${ }^{35}$ Samples were then washed thrice with serum-free media (5 min wash) and fixed. C6-NPs localization to these different organelles was then assessed via a confocal laser scanning microscopy (CLSM; TCS SPE II, Leica, Germany).

\section{Assessment of Cur-NPs Biodistribution Within Zebrafish}

Zebrafish (Danio rerio) were obtained from the China Zebrafish Resource Center (Wuhan, China), and housed 
under standard conditions with a $14 \mathrm{~h} / 10 \mathrm{~h}$ light/dark cycle. After reaching maturity, male and female zebrafish were added to a $1 \mathrm{~L}$ tank at a 1:2 ratio with a screen separating them overnight, after which fertilized embryos were collected. In order to block the formation of pigment, 1-phenyl-2- thiourea was used. Cur-NP biodistribution profiles were assessed following oral uptake by incubating these embryos ( 7 days post-fertilization [dpf]) in a solution of C6-NPs at a $400 \mathrm{ng} / \mathrm{mL}$ C6 concentration for 15 or 60 min. Following treatment, C6-NP biodistribution was assessed via fluorescent microscopy (DMi8, Leica, Germany).

\section{Analysis of Cur-NPs Pharmacokinetics}

Male C57BL/6 mice (8 weeks old) were housed under controlled conditions $\left(25^{\circ} \mathrm{C}, 55 \%\right.$ relative humidity, 12 $\mathrm{h}$ light/dark cycle) with free food and water access. Animals were orally administered Cur-NPs or an equivalent dose of Cur $(5 \mathrm{mg} / \mathrm{kg})$ in $1.5 \mathrm{mg} / \mathrm{mL}$ PVP K90 (controls). At 1, 2, 4, and $8 \mathrm{~h}$ post-treatment, blood samples were collected from the tail vein of these animals (4/time point). Blood was centrifuged for $10 \mathrm{~min}$ at $3500 \mathrm{rpm}$, and supernatant serum samples were collected for analysis. At these same time points, mice were euthanized via anesthetization and major organs were collected from 4 animals per time point. Levels of Cur in murine plasma and organ samples were then assessed via LC-MS/MS ${ }^{36}$.

\section{Establishment of $\mathrm{ICH}$ Mouse Model}

Male C57BL/6 mice (8-10 weeks old) were obtained from the Experimental Animal Center of Guangzhou University of Chinese Medicine (Guangzhou, China). A murine ICH model was established as described previously. ${ }^{37}$ Briefly, mice were anesthetized and placed in a prone position with head stabilization in a stereotaxic frame. A dental drill was then utilized to generate a $1 \mathrm{~mm}$ burr hole at $2.0 \mathrm{~mm}$ to the lateral right of the bregma and $3.5 \mathrm{~mm}$ deep of the brain. Next, acute ICH was induced by slowly injecting $0.1 \mathrm{U}$ of type IV collagenase into this hole. Control animals were instead injected with an identical volume of saline solution. The Animal Ethics Committee of Guangzhou University of Chinese Medicine approved these experiments, which were performed in accordance with the guidelines pertaining to experimental animal care and use in China.

\section{Drug Treatment}

For experimental treatment, mice were randomized into Control, ICH, Cur, and Cur-NP groups (n=6/group). Cur and Cur-NP solutions were orally administered to appropriate animals via gavage at $2 \mathrm{~h}$ post-ICH injury induction. These drugs were administered twice per day for 3 days post-ICH induction at a $20 \mathrm{mg} / \mathrm{kg} / \mathrm{day}$ dose, with equivalent volumes of saline being administered to mice in the other treatment groups.

\section{Behavioral Testing}

The effects of Cur-NP or Cur treatment on motor activity in ICH model mice were evaluated using rotarod and pole climbing assays. For rotarod tests, animals were placed on a rod rotating at $20 \mathrm{rpm}$ for $120 \mathrm{~s}$, with the number of falls and the latency to fall during this period being recorded. For pole climbing tests, mice were positioned atop a pole (50 $\mathrm{cm}$ high, $0.9 \mathrm{~cm}$ in diameter) and allowed to climb down it for $5 \mathrm{~min}$ without any other interference. Both the time it took for mice to turn downwards (T-turn) and the total time to reach the bottom (T-total) were recorded.

\section{Hematoma Analyses}

Following behavioral testing, mice were euthanized and whole brain tissue sections were excised and cut into $1 \mathrm{~mm}$-thick sections following PBS perfusion. An Epson Perfection V370 photo scanner (Epson China, Beijing, China) was used to acquire images of these tissue sections, and the percentage of hematoma volume [(hematoma volume/hemispheric brain volume) $\times 100$ ] was measured using ImageJ.

\section{Paraffin-Embedded Brain Tissue Preparation}

After euthanasia, mice were transcardially perfused with PBS and then with 4\% PFA. The whole brain was then collected from each animal and transferred into a 4\% PFA solution. After fixation, these brain samples were paraffin-embedded and sliced to yield $5 \mu \mathrm{m}$ sections for subsequent analysis.

\section{Hematoxylin and Eosin (H\&E) Staining}

The impact of Cur-NP treatment on neuron loss in the perihematomal region in brain samples from ICH model mice was assessed via H\&E staining. Briefly, tissue sections were deparaffinized with xylene, rehydrated with an ethanol gradient, and stained for 5 min with hematoxylin and for $1 \mathrm{~min}$ with eosin. Sections were then dehydrated with an ethanol gradient, cleared using xylene, and mounted using neutral gum. Samples were then visualized via optical microscopy (DMi8, Leica, Germany). 


\section{Nissl Staining}

The effects of Cur-NP treatment on neuronal degeneration within the perihematomal region in ICH model mice were evaluated via Nissl staining. Briefly, tissue sections were deparaffinized with xylene, rehydrated using an ethanol gradient, and stained for 10 min using $1 \%$ toluidine blue. They were then dehydrated with an ethanol gradient and mounted using neutral gum. Nissl bodies in these samples were then visualized via optical microscopy (DMi8, Leica, Germany).

\section{Prussian Blue Staining}

Iron accumulation within the perihematomal region was evaluated via Prussian blue staining. Briefly, tissue sections were deparaffinized with xylene, rehydrated with an ethanol gradient, and treated for $15 \mathrm{~min}$ with Perls Prussian Blue stain and for $30 \mathrm{~s}$ with hematoxylin. An ethanol gradient was then used to dehydrate these samples, which were subsequently treated with dimethyl benzene to transparency and mounted onto neutral resin cover slides. Sections were then imaged via optical microscopy (DMi8, Leica, Germany).

\section{Immunofluorescent Staining}

For GPX4 staining, $30 \mu \mathrm{m}$-thick sections of frozen brain tissue were permeabilized with $0.1 \%$ Triton $\mathrm{X}-100$, after which they were blocked at room temperature in goat serum and then probed overnight with anti-GPX4 at $4^{\circ} \mathrm{C}$. Following an additional $1 \mathrm{~h}$ incubation with goat antirabbit IgG (Alexa Fluor 594) while protected from light, DAPI was used to stain cell nuclei, and cells were imaged via fluorescence microscopy (DMI8, Leica, Germany), with positive cells being stained in 3-4 different fields of view per sample.

\section{Cell Viability Assay}

The impact of Cur-NP treatment on cellular viability was assessed via an MTT assay approach. Briefly, HT22 cells were treated with erastin for $24 \mathrm{~h}$ together with Cur-NPs or other experimental treatments. Next, the media in each well was replaced with $90 \mu \mathrm{L}$ of DMEM containing 10 $\mu \mathrm{L}$ of MTT solution, and cells were incubated for an additional $4 \mathrm{~h}$. The supernatants in each well were then discarded and replaced with $150 \mu \mathrm{L}$ of DMSO. After shaking for 10 min using an oscillator, absorbance at 490 $\mathrm{nm}$ in each well was measured with a plate reader
(Multiskan FC, Thermo Scientific, USA) to gauge cell viability.

\section{Live/Dead Cell Staining Assay}

HT22 cells that had been treated with erastin were treated with appropriate concentrations of Cur-NPs or other drugs for $24 \mathrm{~h}$, after which supernatants were replaced with 2 $\mu \mathrm{M}$ of calcein $\mathrm{AM}$ and $8 \mu \mathrm{M}$ of PI for 30 min while protected from light. Cells were then imaged via fluorescence microscopy (DMi8, Leica, Germany).

\section{Annexin V-FITC/PI Assay}

HT22 cells treated with different administration staining with Annexin V and PI was carried out using an Annexin V-FITC/PI Apoptosis Detection kit (KeyGEN Biotech, Nanjing, China). Flow cytometry was performed to detect the apoptosis of HT22 cells. Data were analyzed with CytExpert analysis software.

\section{DCF Fluorescence Staining}

DCF fluorescence staining was performed to investigate the intracellular ROS level in differentially treated HT22 cells and the fluorescence intensity of DCF analysis was carried out by Image J (NIH software).

\section{Detection of ROS}

DHE is a cell-permeative dye that is oxidized to fluorescent ethidium bromide by superoxides and intercalates into DNA, which was used to detect the ROS levels in differentially treated HT22 cells in this research. The ROSpositive cells were identified using a fluorescent microscope (DMI8, Leica, Germany).

\section{Quantitative Real-Time Polymerase Chain Reaction (RT -qPCR)}

Total RNA was extracted from the perihematoma brain tissues of the samples in each group using the RNAiso Plus reagent (Takara, Beijing, China). Templates of cDNA were prepared using the PrimeScript ${ }^{\mathrm{TM}}$ RT Master Mix (Takara, Beijing, China) by reverse transcription. The templates were then diluted in the ratio of $1: 3$ and subjected to amplification on a real-time fluorescence quantitative PCR instrument (ABI, California, United States). Amplification of each sample was repeated thrice and the average value was used to calculate the relative content of the product. GAPDH was utilized as the housekeeping gene. The relative mRNA concentrations were measured 
by $\mathrm{E}=2^{-\Delta \Delta \mathrm{Ct}}$, and the key threshold cycle $(\mathrm{CT})$ value was examined in each reaction.

\section{Statistical Analysis}

Data are expressed as the means \pm standard deviation (SD) and were assessed via one-way analysis of variance (ANOVA) and two-tailed Student's $t$ test with $P<0.05$ as the significance threshold.

\section{Results and Discussion}

\section{Cur-NPs Characterization}

An antisolvent precipitation approach was used to prepare Cur-NPs for the present study (Figure 1A). The resultant particles were spherical in shape and 70-100 $\mathrm{nm}$ in diameter when assessed via TEM (Figure 1B). Dynamic light scattering (DLS) analyses indicated these particles to be $127.31 \pm 2.73 \mathrm{~nm}$ in diameter, with a PDI of $0.21 \pm 0.01$ (Figure 1C), and a zeta potential of -0.25 $\pm 0.02 \mathrm{mV}$ (Figure 1D). XRD analyses did not reveal the presence of distinctive free Cur peaks at $17^{\circ}$ in CurNPs, likely because these peaks were masked by the larger percentage of Cur-NPs in the resultant preparations (Figure 1E). Furthermore, the size, charge and surface modification of C6-NPs were evaluated in this study, which were the main factors that affect the cellular uptake amount of NPs. ${ }^{38,39}$ The results showed that C6-NPs had a similar size and zeta potential as CurNPs.

\section{Assessment of the Uptake and Distribution of NPs by Cells in vitro}

The uptake of NPs by MDCK cells was found to occur in a time and dose-dependent manner (Figure 2A and C). To elucidate the mechanistic basis for such uptake, MDCK cells were treated with different endocytic inhibitors, of which $\mathrm{CPZ}$, HS, and $\mathrm{M} \beta \mathrm{CD}$ had the most profound suppressive impact on NPs endocytosis. These results strongly suggested that a number of nonspecific endocytic mechanisms can facilitate NPs uptake (Figure 2B and D). We then examined the distribution of C6-NPs within cells in vitro, revealing them to be primarily distributed within the ER and lysosomes, which can control drug bioavailability (Figure 2E and F). Mitochondria are critical regulators of energy metabolism and programmed cell death pathways. Mitochondria are also the major site for the production of reactive oxygen species which make this organelle more susceptible to oxidative damage and impairments of mitochondrial functions. It was reported that Curcumin could reduce oxidative damage through increasing reduced glutathione and preventing membrane permeability transition in isolated brain mitochondria. ${ }^{40}$ Notably, NPs system could provide a remarkable improvement in the drug therapeutic index through facilitating antioxidant drugs import into mitochondria. ${ }^{41}$ In this study, NPs significantly colocalized with the mitochondria in treated cells, suggesting that they facilitated the entry of Cur into the mitochondria $^{42}$ (Figure 2G).

\section{Analysis of the in vivo Biodistribution Profiles of NPs}

To explore patterns of in vivo NPs biodistribution following the oral administration of these particles, zebrafish larvae were incubated in the presence of free C6 or C6NPs for 15 or $60 \mathrm{~min}$. Significant time-dependent increases in fluorescent signal were observed in the brain and intestines of zebrafish in the C6-NPs group over this treatment period, whereas negligible fluorescence was evident in the free C6 group. This suggested that these NPs are able to cross the BBB, and are thus ideally suited as a drug delivery approach for enhancing brain accumulation. These zebrafish also exhibited the accumulation of C6-NPs within their eyes, indicating that these NPs can also cross the blood-retinal barrier. In light of these findings, we concluded that Cur-NPs can enhance the absorption of Cur and the accumulation of this drug within the brain (Figure 3A and B). In pharmacokinetic analyses, Cur-NPs concentrations in the plasma of C57BL/6 mice were significantly higher than those for mice administered free Cur (Figure 4A). These results provided robust evidence for the ability of Cur-loaded NPs to enhance the systemic circulation of this drug owing to the ideal size and surface properties of these particles. Cur concentrations in the brains of Cur-NPs-treated mice were significantly higher than in control animals and peaked at 8 $\mathrm{h}$ post-treatment, consistent with the sustained release of Cur from these NPs in the brain, thus enhancing its accumulation therein (Figure 4B). Cur-NPs concentrations in samples of heart, liver, spleen, lung, and kidney tissues from treated mice were also elevated relative to control animals, consistent with the sustained release of Cur from Cur-NPs and its high plasma exposure rate (Figure $4 \mathrm{C}-\mathrm{G}){ }^{43}$ 
A

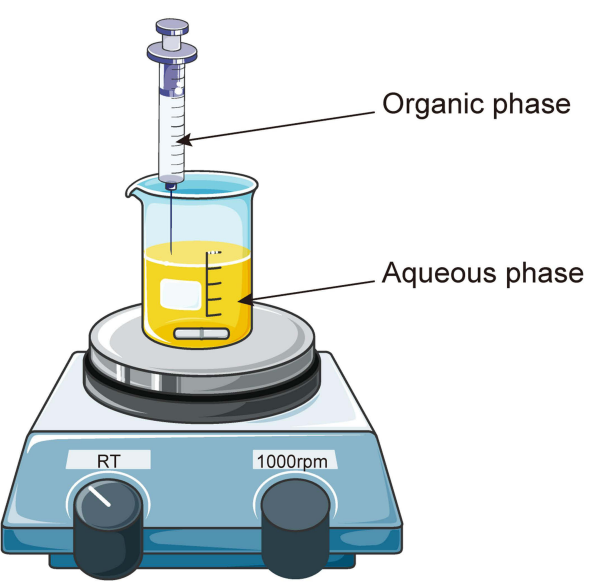

C

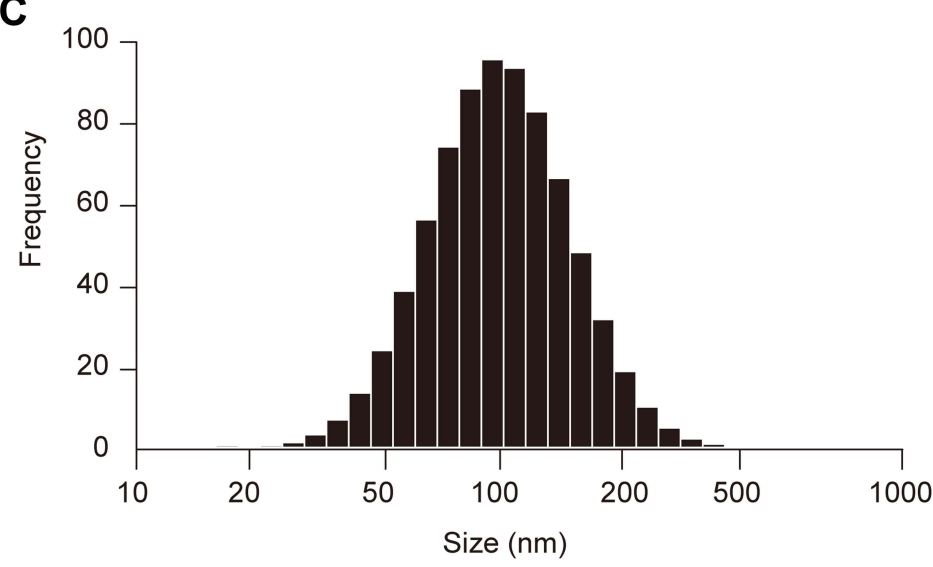

E

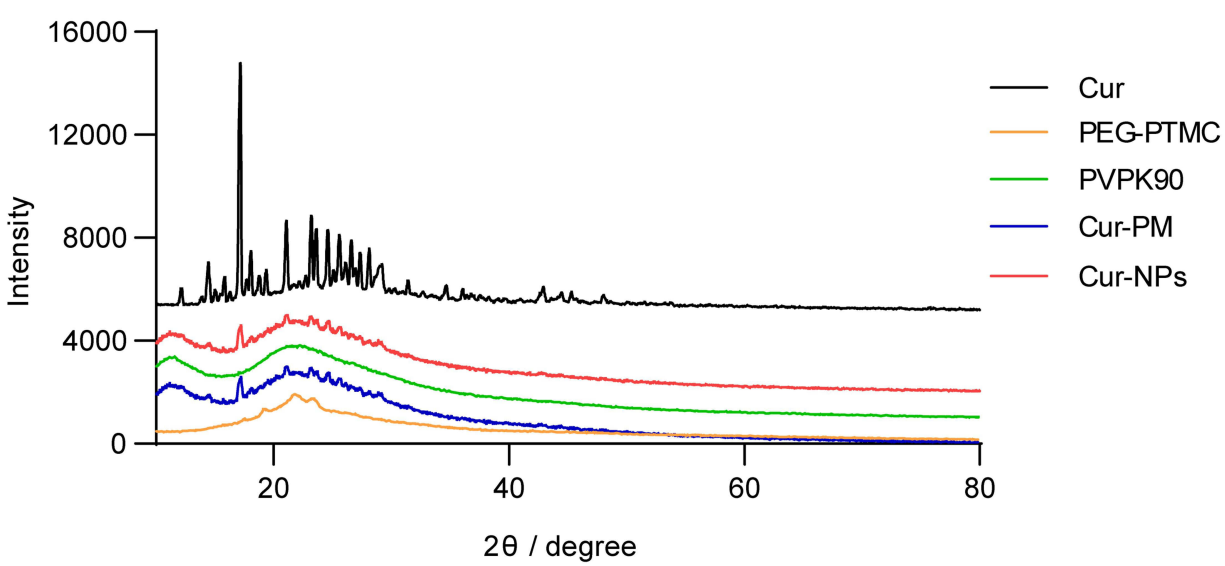

B

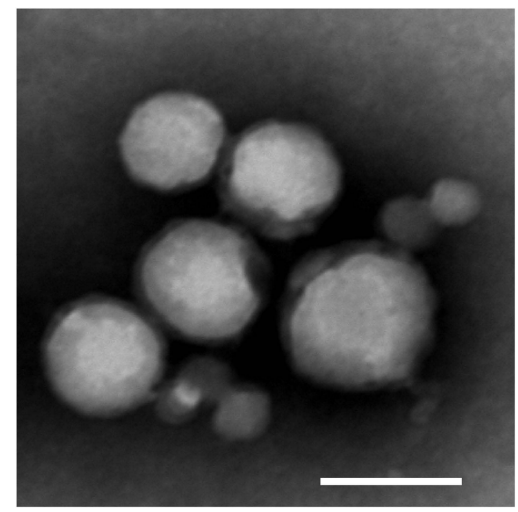

D

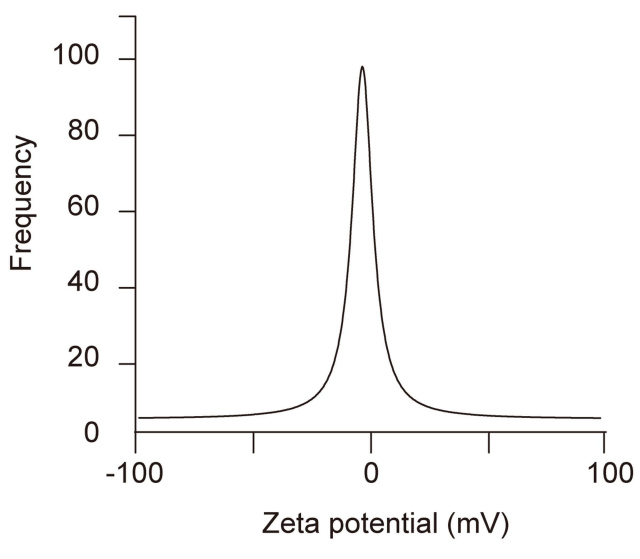

Figure I Cur-NPs characterization. (A) Cur-NPs preparation. (B) TEM imaging results. Scale bar: 100 nm. (C) Size distributions. (D) Zeta potential values. (E) XRD plots for Cur, PEG-PTMC, PVP K90, Cur-PM (a physical mixture of PVP K90 and Cur), and Cur-NPs.

\section{Cur-NPs Suppressed ICH-Induced Behavioral Deficits in Mice}

When ICH model mice were evaluated via a rotarod test, they exhibited a significantly shorter fall latency relative to control animals, while this latency was significantly increased in Cur-NPs treated mice. Cur-NPs also significantly reduced the number of falls for these ICH model mice (Figure 5A). Similarly, in a pole climbing test, ICH resulted in a marked increase in both the T-turn and T-total values, while Cur-NPs treatment was sufficient to reverse 
A
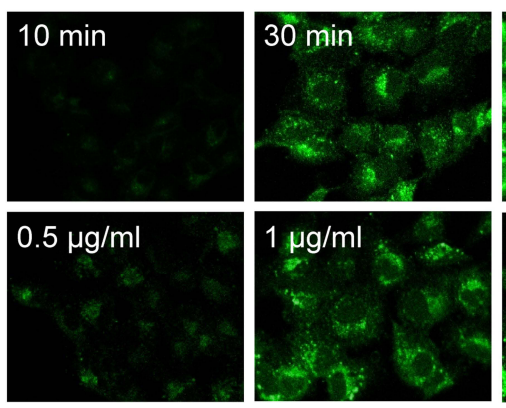

C

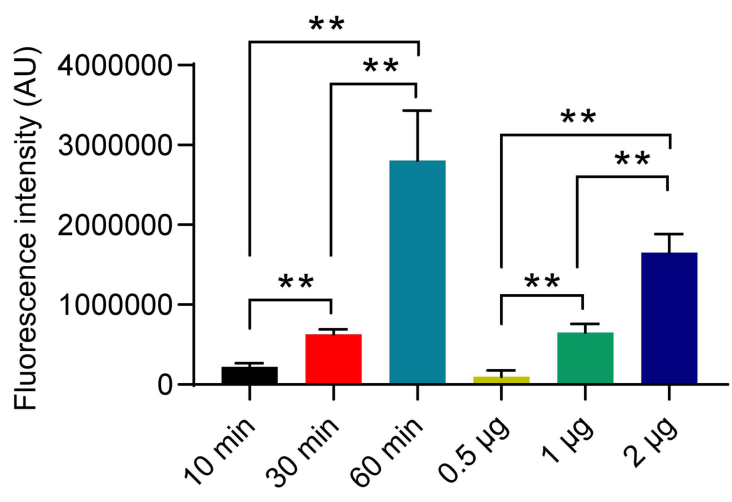

E
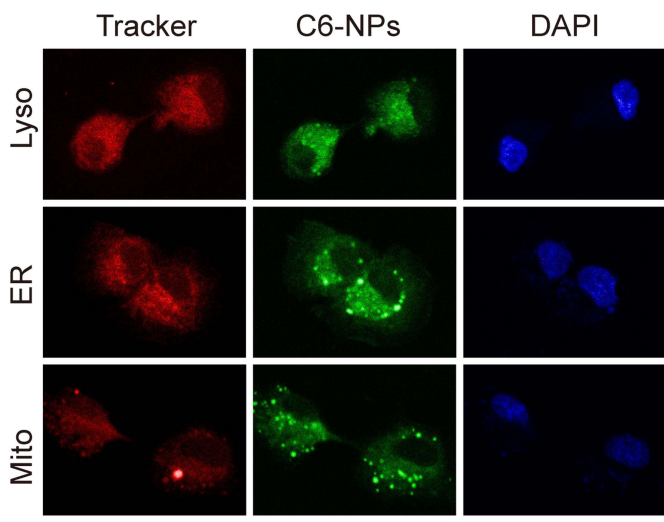
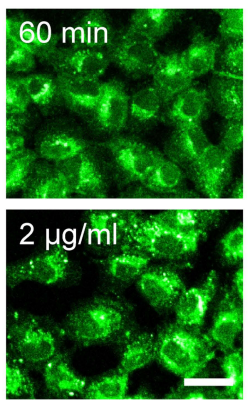

B
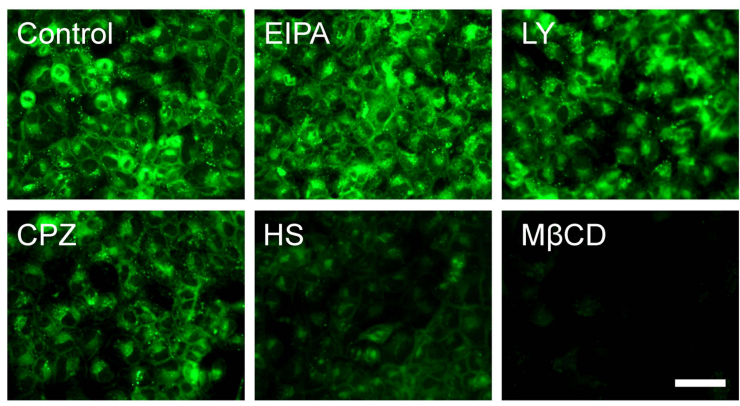

$M \beta C D$

D

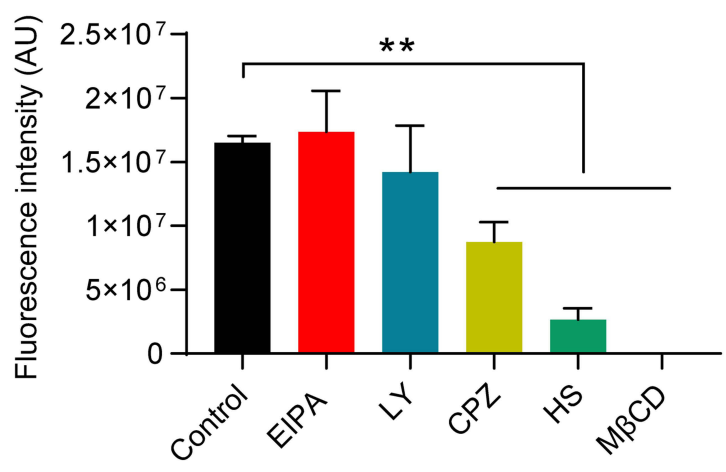

F
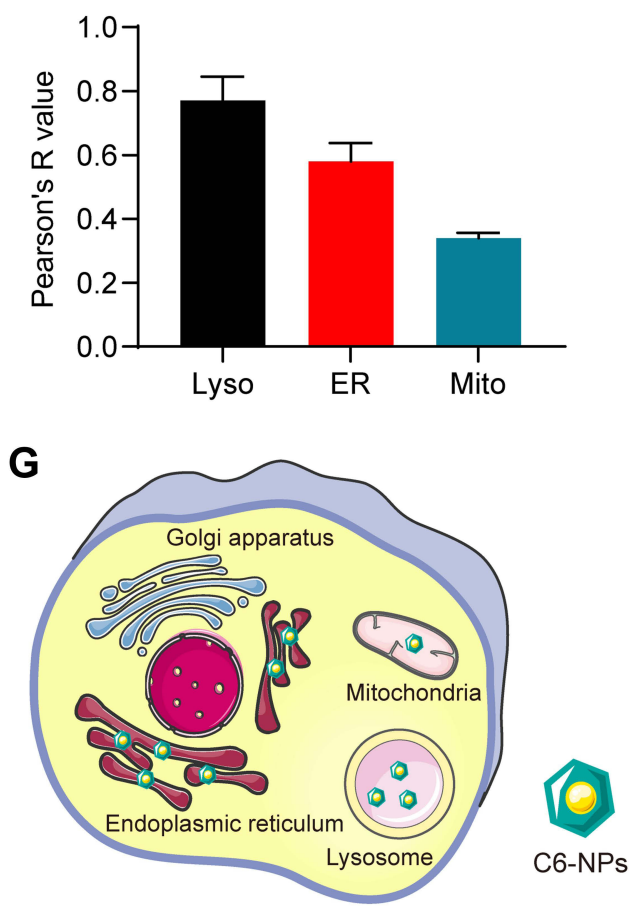

Figure 2 NPs cellular uptake and subcellular localization. (A) C6-NPs exhibited time- and dose-dependent uptake by MDCK cells. Scale bar: 50 $\mu$ m. (B) Fluorescent images of endocytic uptake of C6-NPs by MDCK cells. Scale bar: $50 \mu \mathrm{m}$. (C) The quantitative statistical chart of fluorescence intensity in MDCK cells with different time points and doses. (D) The quantitative statistical chart of fluorescence intensity in MDCK cells with treated with different endocytic uptake inhibitors. (E) C6-NPs distributions in cells stained with the indicated organelle tracking dyes. Scale bar: $25 \mu \mathrm{m}$. (F) The quantitative statistical chart of Pearson value of C6-NPs distributions in cells stained with the indicated organelle tracking dyes. (G) An overview of the process of C6-NP cellular uptake. **Indicates $\mathrm{P}<0.01$.

these changes (Figure 5B). Importantly, Cur-NPs achieved significantly better therapeutic efficacy as compared to free Cur with respect to the observed improvements in murine behavior. Together, these results suggested that Cur-NPs treatment can partially overcome ICH-induced behavioral deficits in mice. 
A

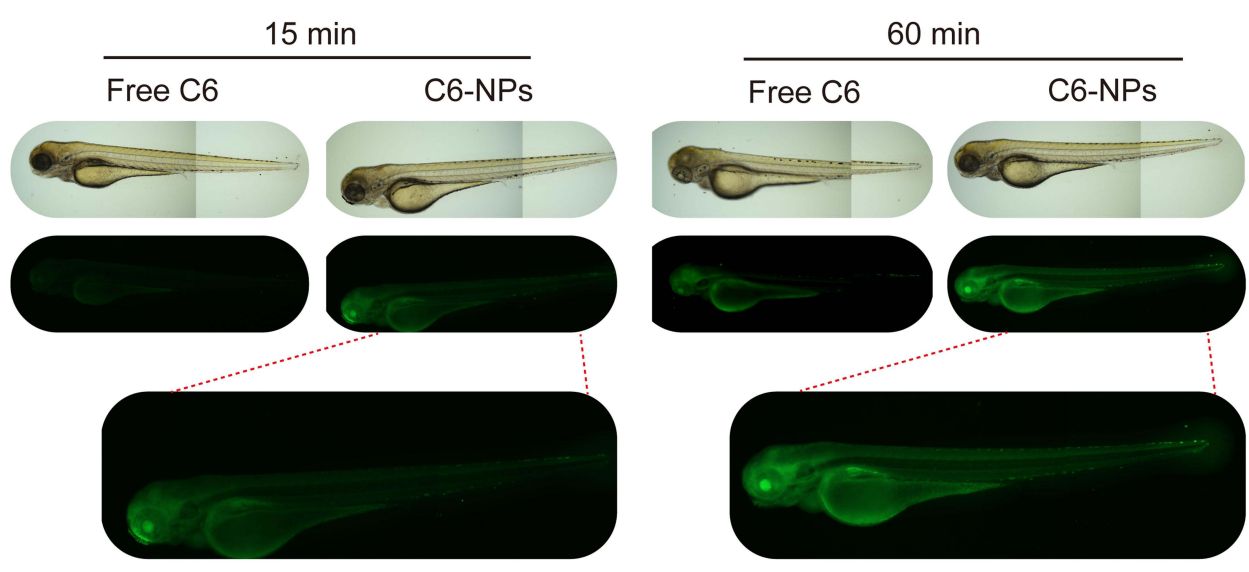

B

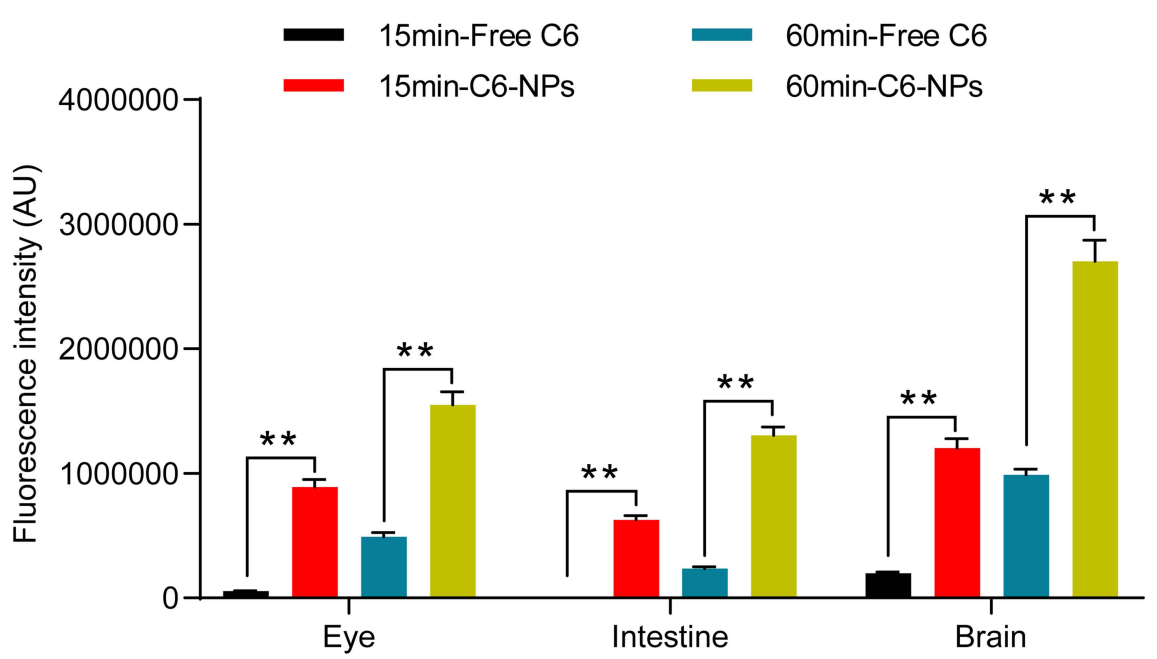

Figure 3 Assessment of the in vivo biodistribution of C6-NPs. (A) Zebrafish larvae (7 dpf) were treated with C6-NPs (400 ng/mL) for 15 or $60 \mathrm{~min}$. Scale bar: $100 \mu \mathrm{m}$. (B) The quantitative statistical chart of fluorescence intensity in zebrafish. **Indicates $\mathrm{P}<0.0 \mathrm{I}$.

\section{Cur-NPs Reduced the Hematoma Volumes in the Murine Brain}

Cur-NPs treatment was associated with a significant decrease in hematoma volume as compared to the ICH and Cur treatment groups (Figure 6A and B). H\&E staining further indicated that ICH was associated with significant perihematomal neuronal loss, whereas Cur-NPs treatment was sufficient to prevent such neuronal damage (Figure 6C).

\section{Cur-NPs Treatment Attenuated the Severity of ICH-Induced Neurological Injury}

ICH model mice exhibited clear perihematomal neuron degeneration in Nissl-stained brain tissue sections, whereas this neuronal damage was markedly attenuated in mice treated with Cur-NPs to a greater extent than was observed in Cur-treated mice. The accumulation of iron within cells in the perihematomal region was assessed in these mice via Prussian Blue staining, which demonstrated that Cur-NPs were associated with a substantial drop in the frequency of Prussian bluepositive cells relative to the ICH model group and the Cur treatment group, consistent with the ability of these Cur-NPs to suppress iron deposition within the brain tissue surrounding the hematoma site. GPX4 is a glutathione peroxidase that functions by detoxifying lipid peroxides, ${ }^{44}$ and it serves as a key ferroptosis marker owing to its ability to suppress intracellular lipid peroxidization. ${ }^{45,46}$ We found that the administration of Cur-NPs was associated with a corresponding 
A B

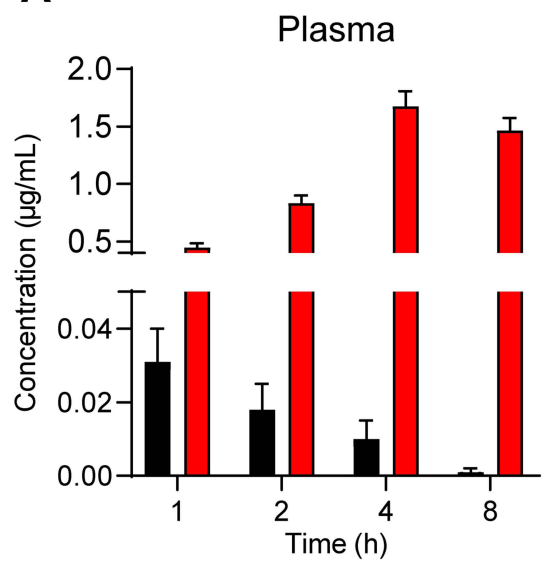

D

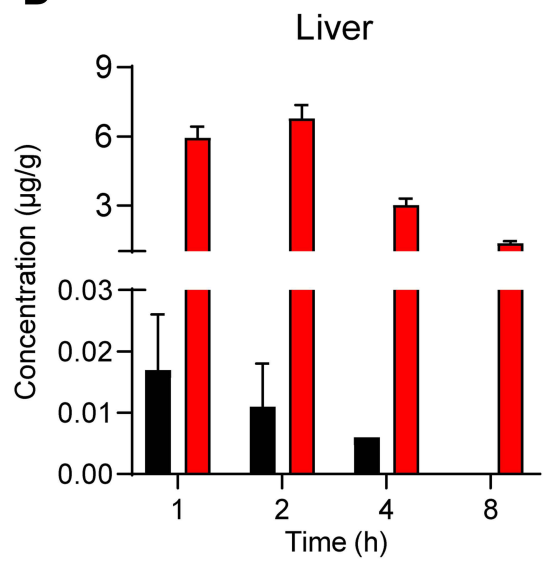

G

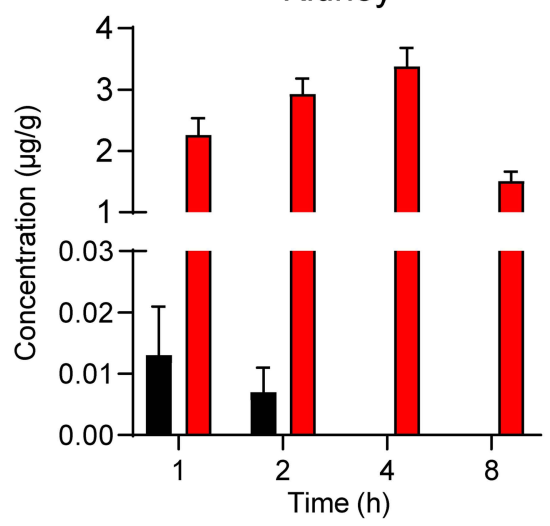

B C

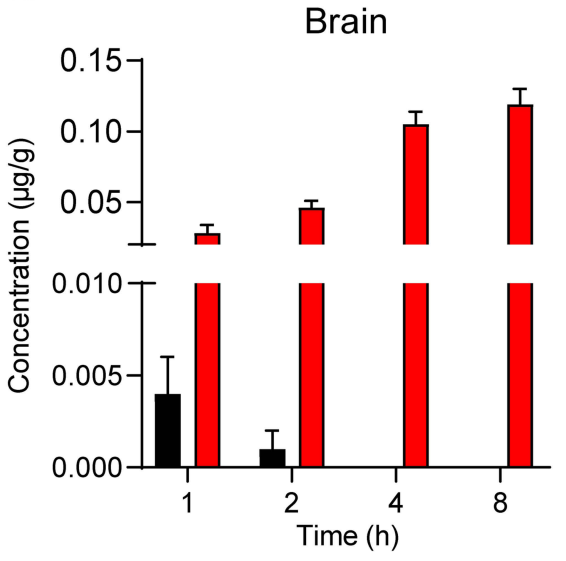

$\mathbf{E}$

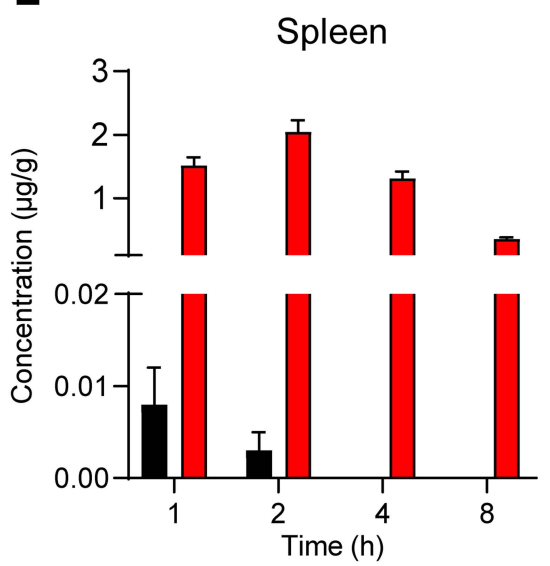

C

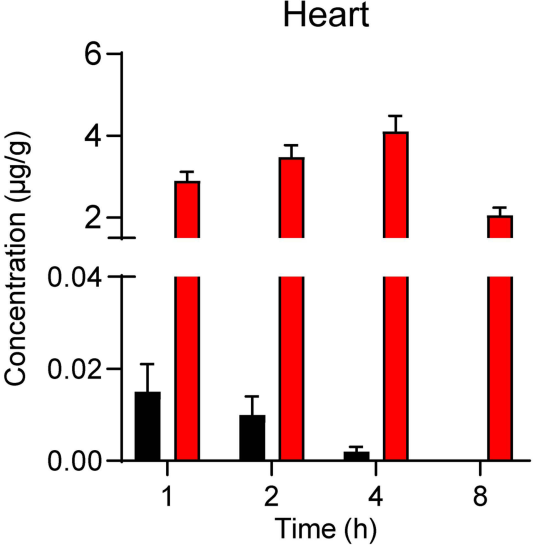

$\mathbf{F}$

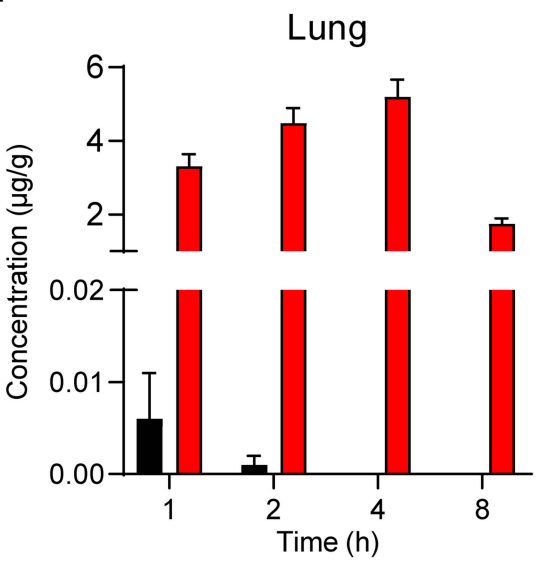

Figure 4 The concentration of Cur-NPs in plasma (A), brain (B), heart (C), liver (D), spleen (E), lungs (F) and kidneys (G) were assessed in treated C57BL/6 mice (means $\pm S D, n=4)$.

increase in perihematomal GPX4 expression in ICH model mice (Figure 7A), thus suggesting that Cur-NPs treatment can significantly attenuate the induction of ferroptosis in this pathological context. Erastin induces ferroptosis through inhibition of the NRF2/
HO-1 pathway. Furthermore, we investigated the effect of Cur-NPs on NRF2/HO-1 pathway, and the results showed that Cur-NPs significantly regulated the expression of HMOX1 (HO-1) and NFE2L2 (NRF2) in ICH model mice (Figure $7 \mathrm{~B}$ and $\mathrm{C}$ ). These findings 
A

Day1
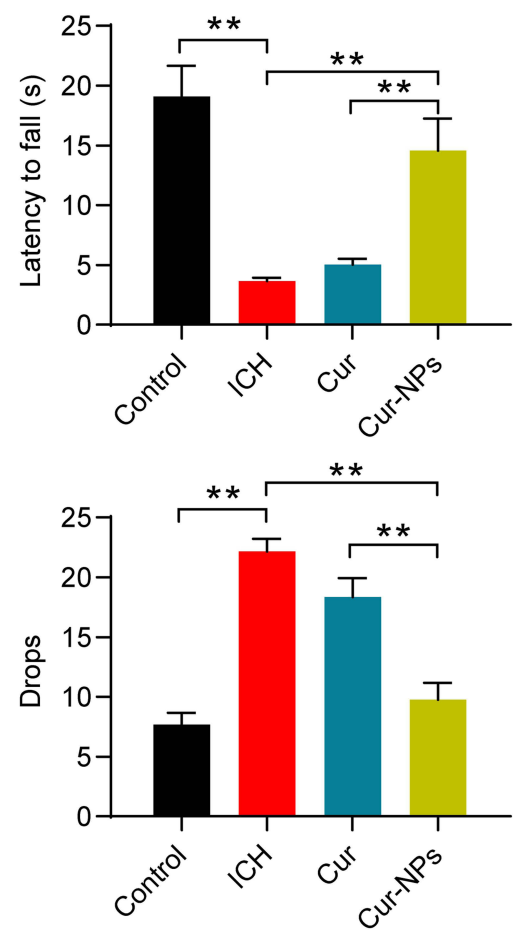

B

Day1
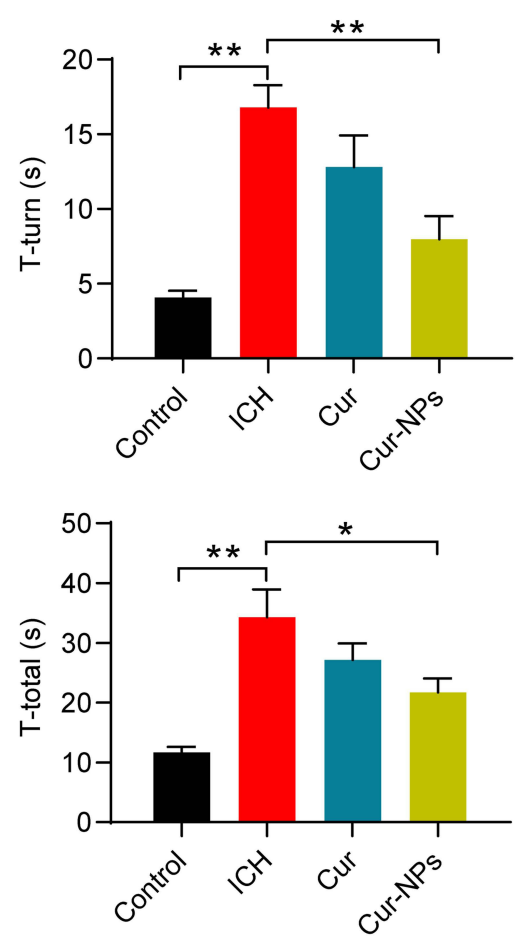

Day2
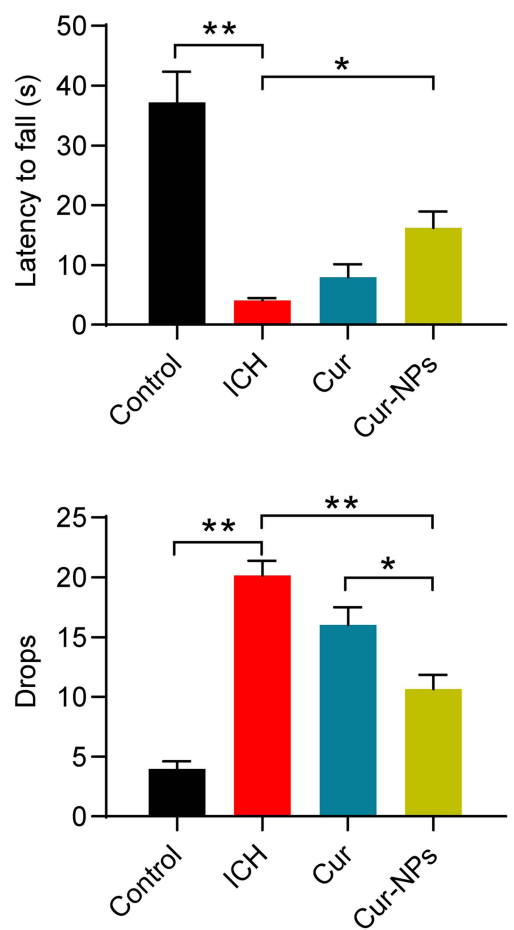

Day2
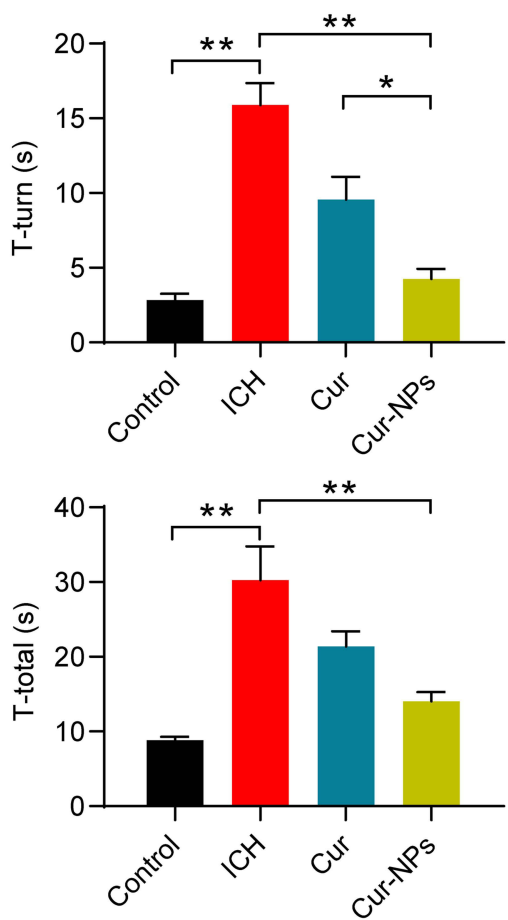

Day3
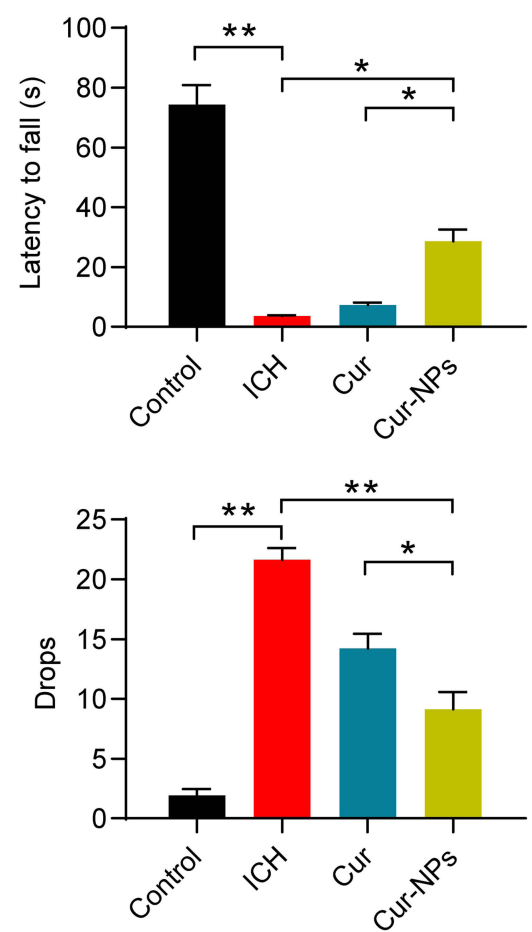

Day3
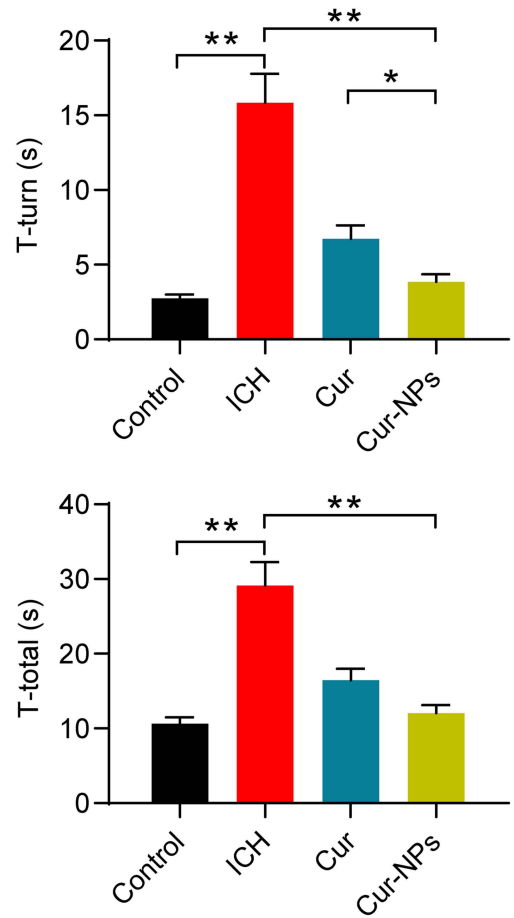

Figure 5 The impact of Cur-NPs on ICH-induced behavioral deficits. (A) Fall latency and numbers of drops in rotarod tests for mice in the indicated groups (means \pm SD, $n=12$ ). (B) T-turn and T-total times for pole tests of mice in the indicated groups (means $\pm S D, n=12$ ). $*$ Indicates $P<0.05$ and $* *$ Indicates $P<0.01$. 

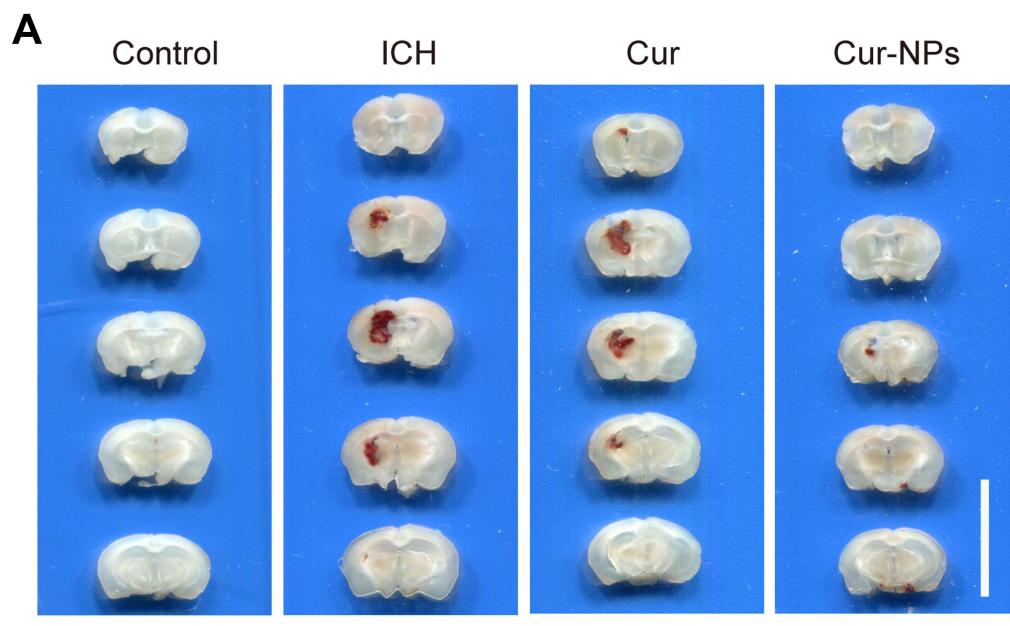

B

C Control

$\mathrm{ICH}$

Cur
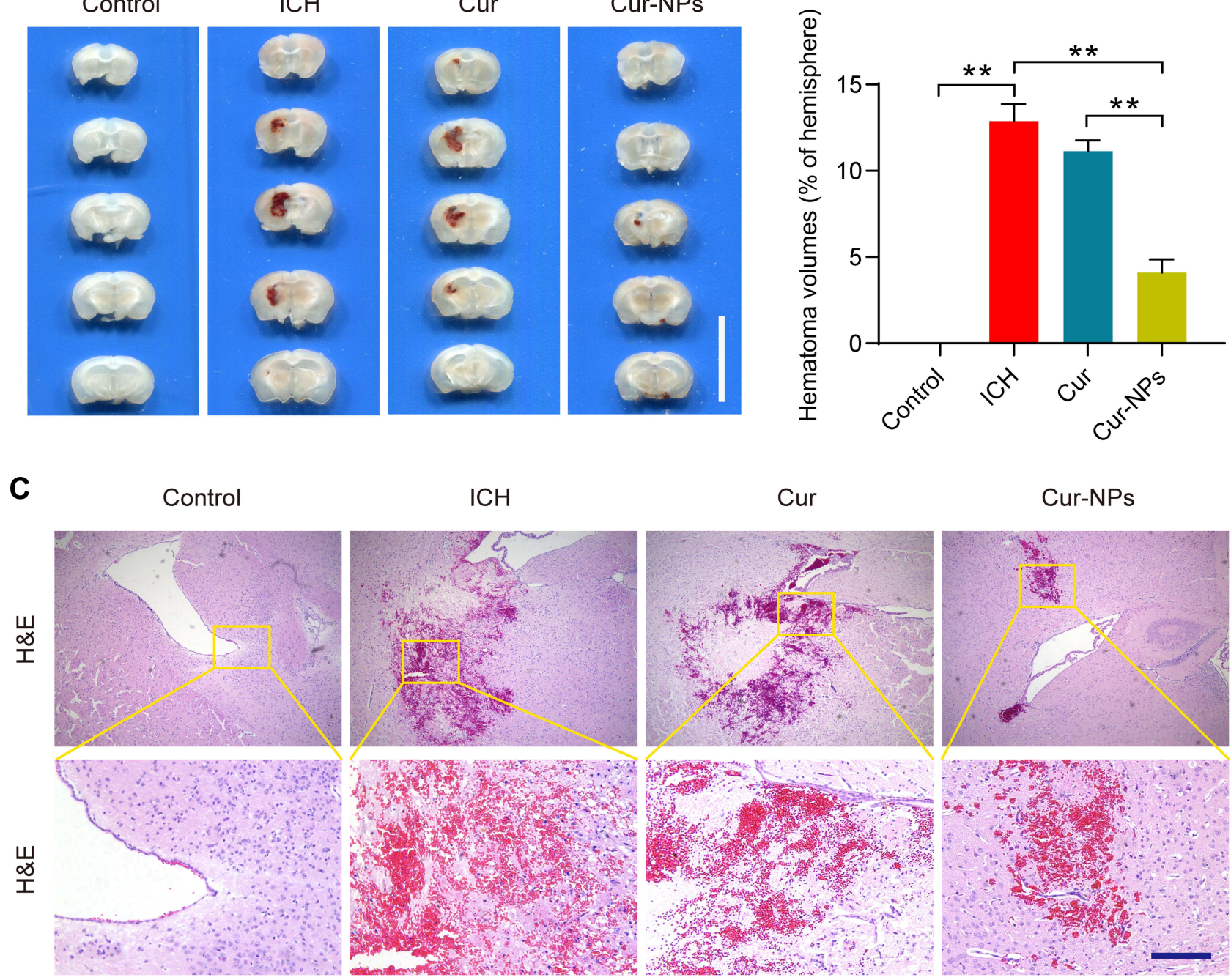

Figure 6 Cur-NPs treatment is associated with a reduction in the hematoma volume in the murine brain. (A) Hematoma images in brain tissue sections from ICH model mice. Scale bar: $10 \mathrm{~mm}$. (B) The percentage of hematoma volume for ICH model mice in the indicated groups $($ means $\pm S D, n=6)$. (C) H\&E stained brain tissues sections from the indicated groups. **Indicates $\mathrm{P}<0.0 \mathrm{I}$.

strongly suggested that Cur-NPs treatment might increase the GPX4 expression by regulating NRF2/HO1 pathway.

\section{Cur-NPs Suppressed Erastin-Induced by HT22 Cell Ferroptosis}

In an MTT assay, Cur-NPs at doses of $20 \mu \mathrm{M}$ or less did not induce any significant toxicity when used to treat cells (Figure 8A). We then explored the anti-ferroptotic activity of these Cur-NPs by applying them together with the known ferroptosis inducer erastin. ${ }^{47}$ In subsequent MTT assays, we found that Cur-NPs were effectively able to enhance the survival of HT22 cells relative to the erastin and Cur-PM treatment groups (Figure 8B), and this enhanced survival was further confirmed through live/dead staining analyses (Figure 8C). Moreover, the results of Annexin V/PI staining showed that Cur-NPs remarkably reduced apoptosis induced by erastin in a dose-dependent manner (Figure 8D). Furthermore, we investigated the effect of Cur-NPs on NRF2/HO-1 pathway and ROS generation. The results showed that Cur-NPs administration significantly inhibited the generation of ROS induced by erastin (Figure 9A-D). Importantly, Cur-NPs effectively regulated the expression levels of HMOX1 and NFE2L2, which indicated that it might inhibit the ROS 
A

Control
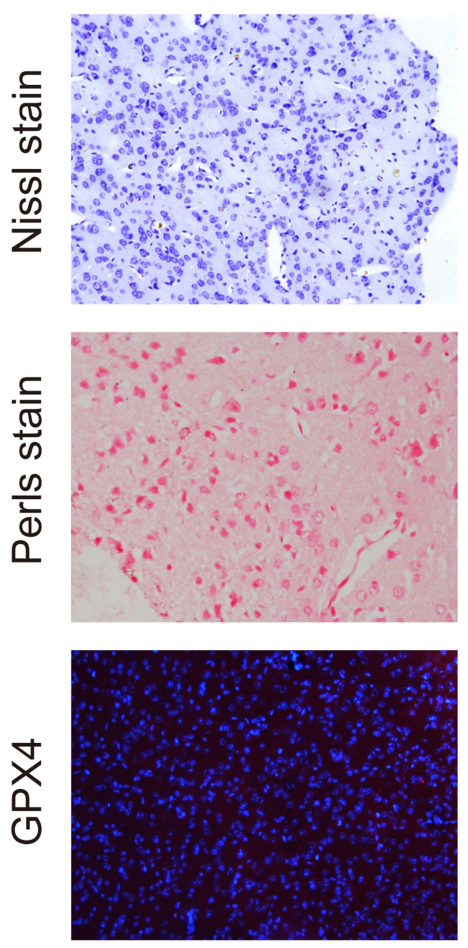

B

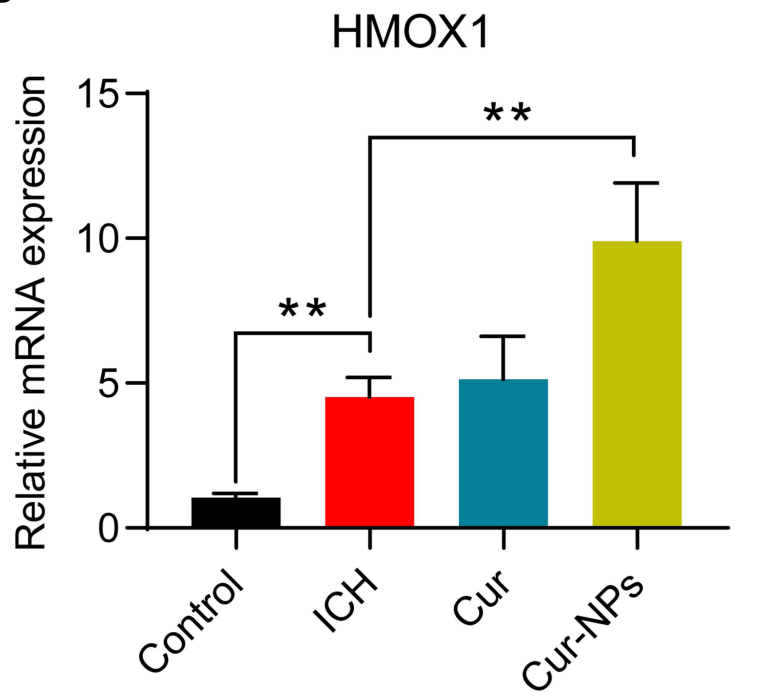

$\mathrm{ICH}$
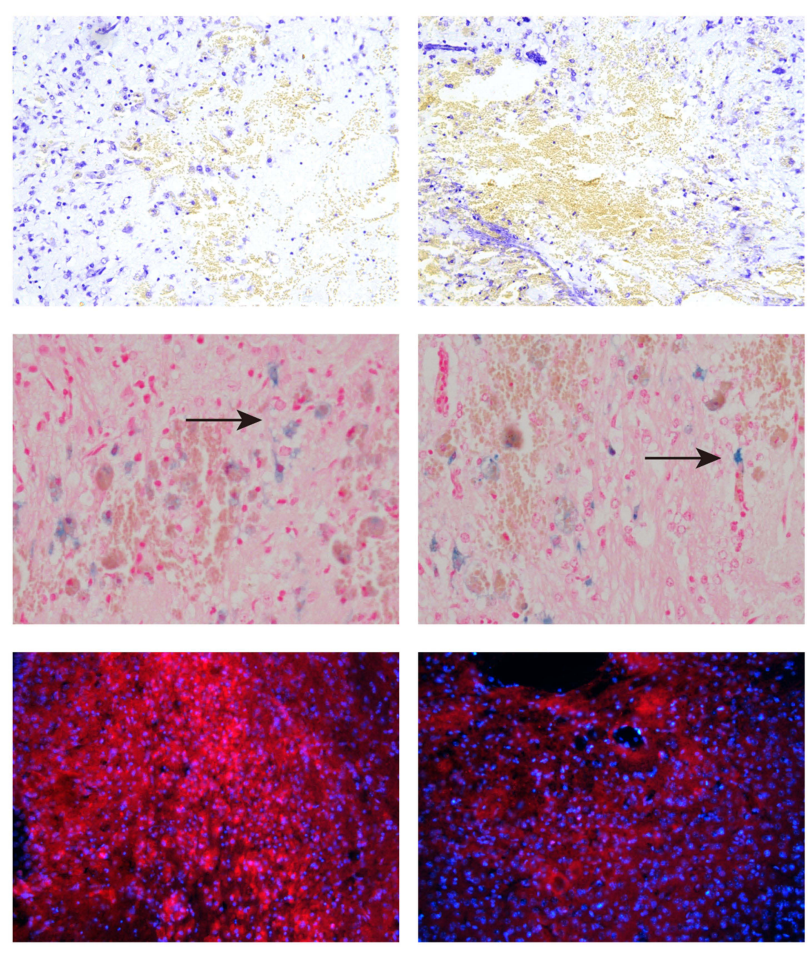

C

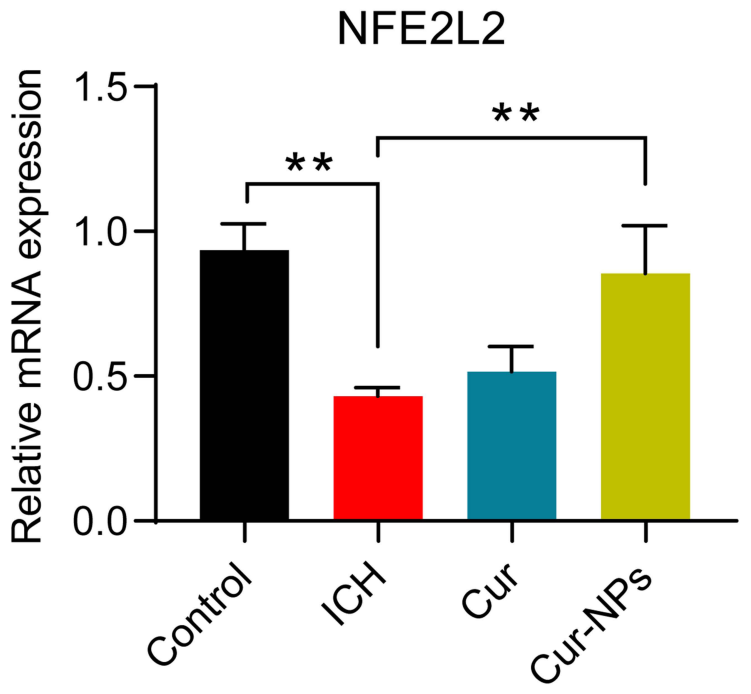

Figure 7 (A) Representative microphotographs of murine brain sections subjected to the indicated treatments and staining regimens. Scale bar: $250 \mu$ m. (B-C) The relative mRNA expression of HMOXI and NFE2L2 in the perihematoma tissue of $\mathrm{ICH}$ mice in different groups. **Indicates $\mathrm{P}<0.01$.

production through regulating the NRF2/HO-1 pathway (Figure 9E and F).

\section{Conclusions}

In this study, an antisolvent precipitation approach was used to prepare Cur-NPs, which were readily taken up by cells through multiple nonspecific endocytic mechanisms, with plasma membrane microcapsules and clathrin-mediated uptake being the dominant internalization modalities. These Cur-NPs primarily accumulated within ER, lysosomes and mitochondria, and were efficiently transported across physiological barriers to enhance the accumulation of 

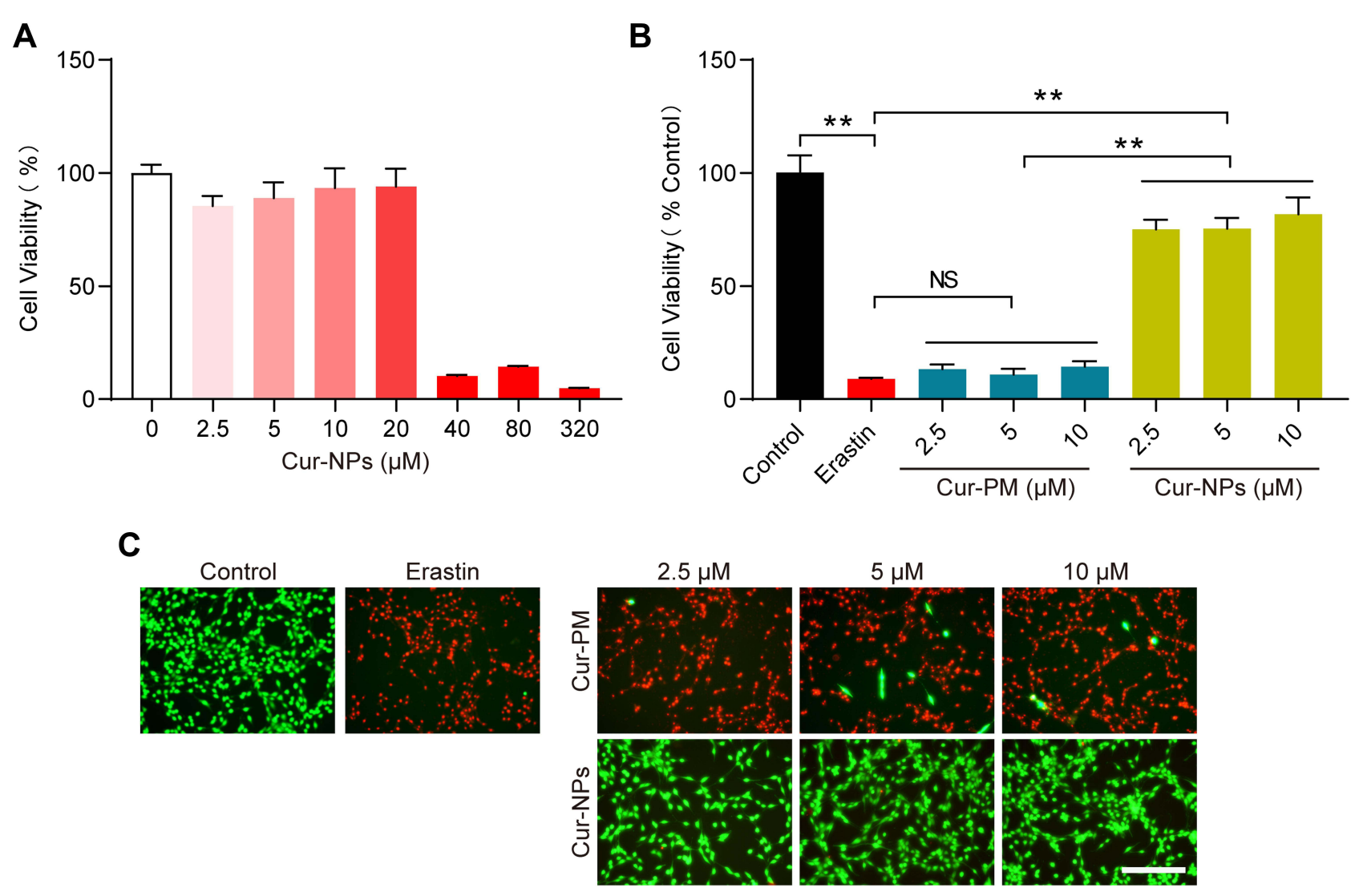

D
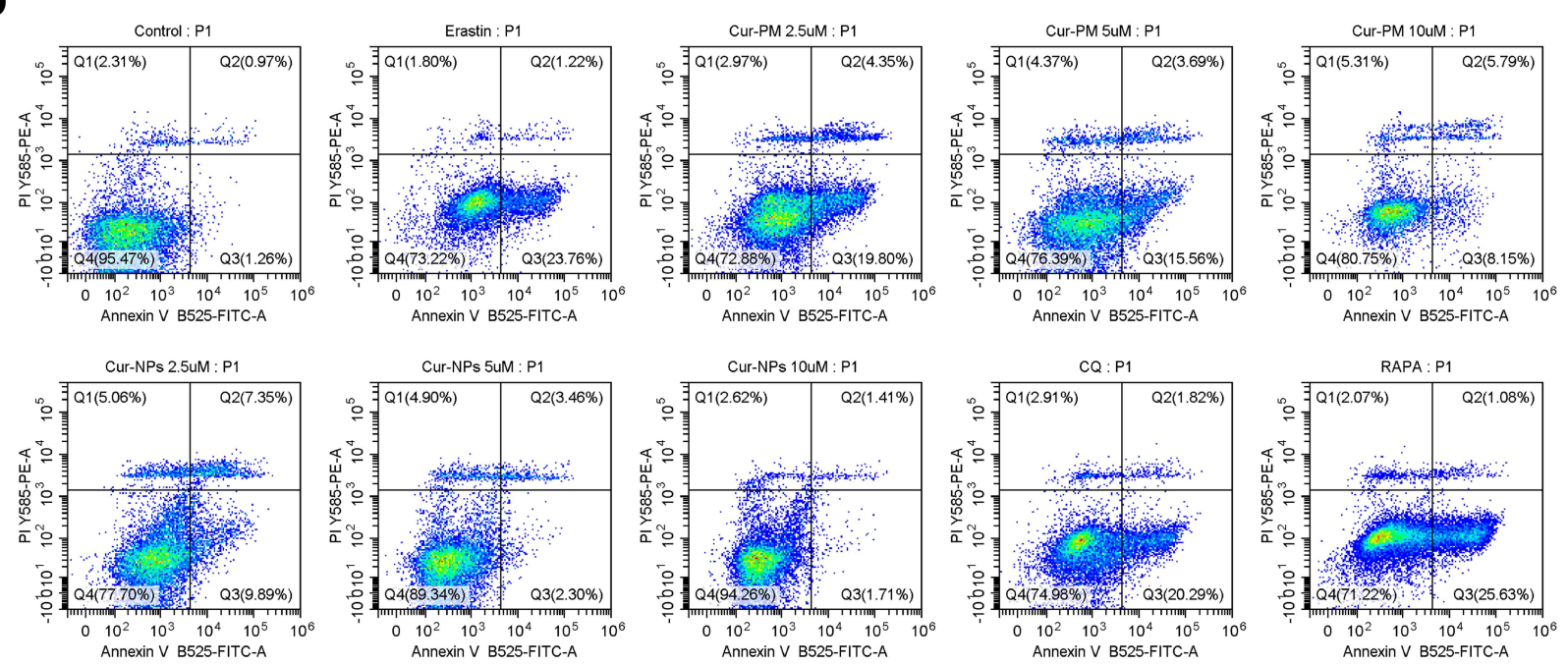

Figure 8 Erastin-induced HT22 cell viability following Cur-NP and Cur-PM treatment. (A) An MTT assay was used to assess the viability of cells treated with different CurNP concentrations. (B) Cells subjected to the indicated treatments were used for an MTT assay. (C) Cells subjected to the indicated treatments underwent Live/dead staining. (D) Flow cytometry dot plots of Annexin V-FITC/PI double staining for the cell apoptosis detection. (Cur-PM, a physical mixture of PVP K90 and Cur). **Indicates $P<0.01$.

Cur within the plasma and brain. More notably, these CurNPs served as an effective treatment for ICH owing to their ability to inhibit ferroptosis. Taken together, Cur-NPs repre- sent a promising tool for enhancing the delivery of Cur to the brain and thereby achieving superior therapeutic outcomes when treating ICH. 
A
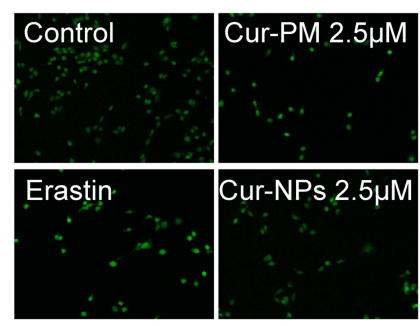

B

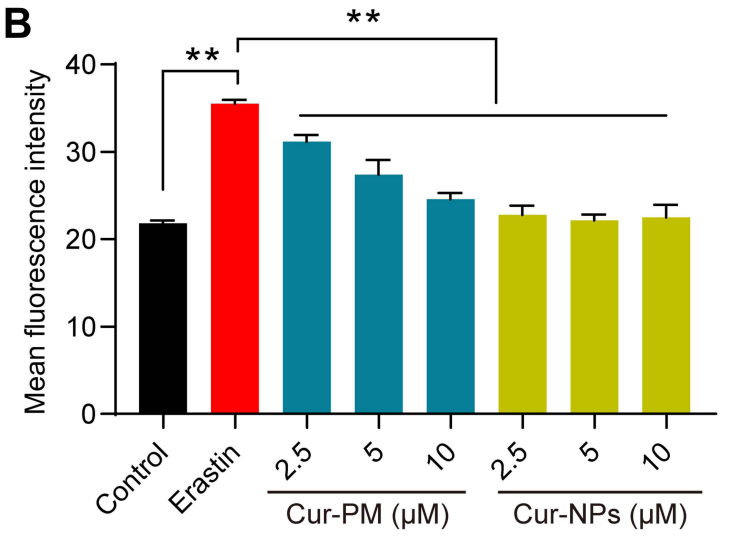

$\mathbf{E}$

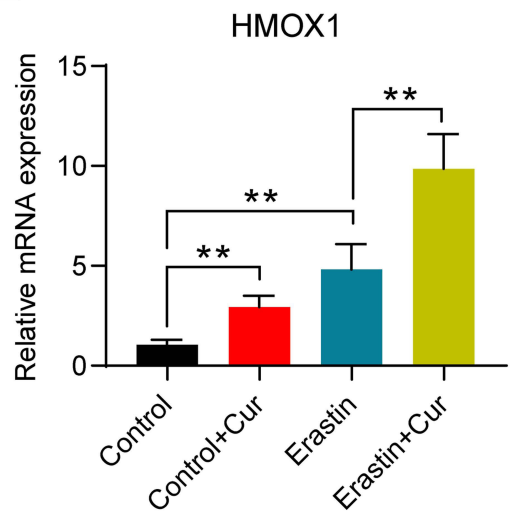

C

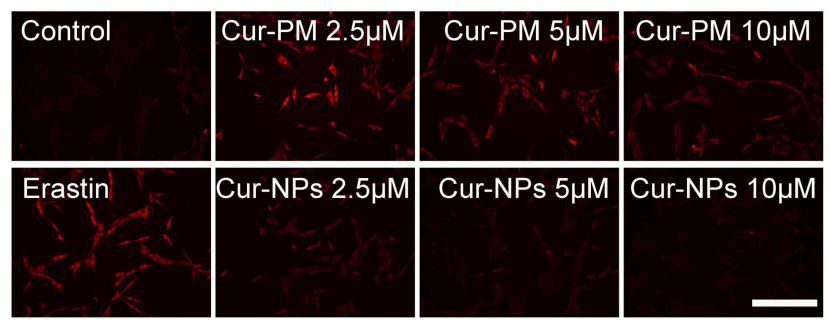

D

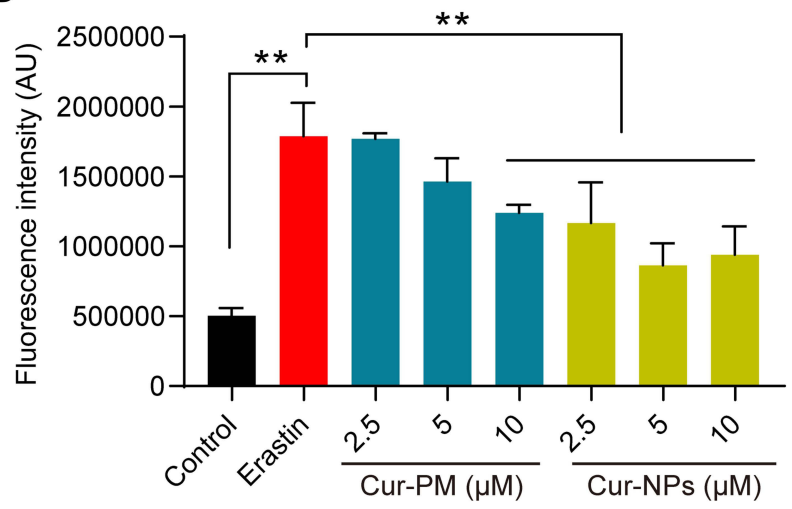

$\mathbf{F}$

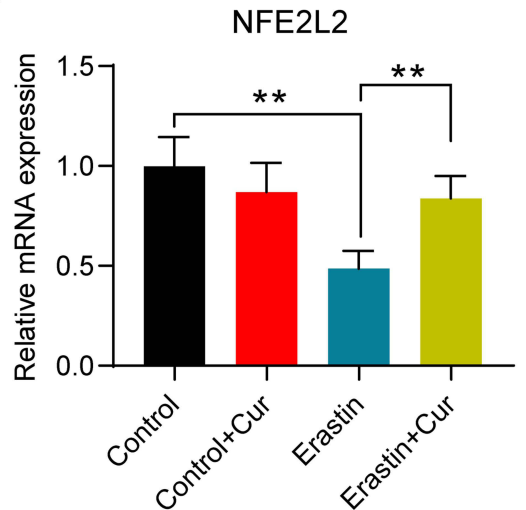

Figure 9 (A) Images of DCF staining in response to the indicated treatments. (B) Statistical analysis of DCF fluorescence intensity in response to the indicated treatments. (C) Images of DHE staining in response to the indicated treatments. (D) Statistical analysis of DHE fluorescence intensity in response to the indicated treatments. (E, F) The relative mRNA expression of HMOXI and NFE2L2 in cells subjected to the indicated treatments. $* *$ Indicates $\mathrm{P}<0.0 \mathrm{I}$.

\section{Credit Author Statement}

All authors made a significant contribution to the work reported, whether that is in the conception, study design, execution, acquisition of data, analysis and interpretation, or in all these areas; took part in drafting, revising or critically reviewing the article; gave final approval of the version to be published; have agreed on the journal to which the article has been submitted; and agree to be accountable for all aspects of the work.

\section{Acknowledgments}

This work was supported by Key laboratory project of colleges and universities in Guangdong province (2019KSYS005), the Guangdong province science and technology plan international cooperation project (2020A0505100052), the Guangdong Basic and Applied Basic Research Foundation (2019B1515120043), the key Project of Basic Research of Shenzhen (JCYJ20200109113603854), and the Guangdong Provincial Natural Science Foundation of China (2018A030310623). 


\section{Disclosure}

The authors declare no potential conflicts of interest in this work.

\section{References}

1. Ding D, Sekar P, Moomaw CJ, et al. Venous Thromboembolism in Patients With Spontaneous Intracerebral Hemorrhage: a Multicenter Study. Neurosurgery. 2019;84:E304-304E310. doi:10.1093/neuros/ nyy333

2. Béjot Y, Blanc C, Delpont B, et al. Increasing early ambulation disability in spontaneous intracerebral hemorrhage survivors. Neurology. 2018;90:e2017-2017e2024. doi:10.1212/WNL.00000000 00005633

3. Zhou SY, Cui GZ, Yan XL, et al. Mechanism of ferroptosis and its relationships with other types of programmed cell death: insights for potential interventions after intracerebral hemorrhage. Front Neurosci. 2020;14:589042. doi:10.3389/fnins.2020.589042

4. Chen B, Chen Z, Liu M, et al. Inhibition of neuronal ferroptosis in the acute phase of intracerebral hemorrhage shows long-term cerebroprotective effects. Brain Res Bull. 2019;153:122-132. doi:10. 1016/j.brainresbull.2019.08.013

5. Stockwell BR, Friedmann Angeli JP, Bayir H, et al. Ferroptosis: a regulated cell death nexus linking metabolism, redox biology, and disease. Cell. 2017;171(2):273-285. doi:10.1016/j.cell.2017.09.021

6. Li Y, Liu Y, Wu P, et al. Inhibition of ferroptosis alleviates early brain injury after subarachnoid hemorrhage in vitro and in vivo via reduction of lipid peroxidation. Cell Mol Neurobiol. 2021;41(2):263-278. doi:10.1007/s10571-020-00850-1

7. Weiland A, Wang Y, Wu W, et al. Ferroptosis and Its Role in Diverse Brain Diseases. Mol Neurobiol. 2019;56(7):4880-4893. doi:10.1007/ s12035-018-1403-3

8. Sun S, Li Y, Zhang H, et al. Neuroendoscopic surgery versus craniotomy for supratentorial hypertensive intracerebral hemorrhage: a systematic review and meta-analysis. World Neurosurg. 2020;134:477-488. doi:10.1016/j.wneu.2019.10.115

9. Hemphill JC 3rd, Amin-Hanjani S. Cerebellar Intracerebral hemorrhage: time for evidence-based treatment. JAMA. 2019;322: 1355-1356. doi:10.1001/jama.2019.14673

10. Kuramatsu JB, Biffi A, Gerner ST, et al. Association of surgical hematoma evacuation vs conservative treatment with functional outcome in patients with cerebellar intracerebral hemorrhage. JAMA. 2019;322:1392-1403. doi:10.1001/jama.2019.13014

11. Holste K, Xia F, Garton H, et al. The role of complement in brain injury following intracerebral hemorrhage: a review. Exp Neurol. 2021;340:113654. doi:10.1016/j.expneurol.2021.113654

12. Feng $C$, Huang $S$, Zhou $X$, et al. Interventional ultrasound assisted early local hemostatic drug therapy in traumatic intracerebral hemorrhage. Ultrasound Med Biol. 2020;46:180-187. doi:10.1016/j. ultrasmedbio.2019.08.021

13. Deng S, Feng S, Wang W, Zhao F, Gong Y. Biomarker and drug target discovery using quantitative proteomics post-intracerebral hemorrhage stroke in the rat brain. $J$ Mol Neurosci. 2018;66:639-648. doi:10.1007/s12031-018-1206-z

14. Wang HL, Zeng H, Xu MB, et al. Efficacy and safety of chinese herbal medicine for primary intracerebral hemorrhage: a systematic review of randomized controlled trials. Front Pharmacol. 2019; 10:1139. doi:10.3389/fphar.2019.01139

15. Dong W, Yang B, Wang L, et al. Curcumin plays neuroprotective roles against traumatic brain injury partly via Nrf2 signaling. Toxicol Appl Pharmacol. 2018;346:28-36. doi:10.1016/j.taap.2018.03.020

16. Abu-Taweel GM. Neurobehavioral protective properties of curcumin against the mercury chloride treated mice offspring. Saudi j Biol Sci. 2019;26:736-743. doi:10.1016/j.sjbs.2018.10.016
17. Zhang J, Tang L, Li GS, Wang J. The anti-inflammatory effects of curcumin on renal ischemia-reperfusion injury in rats. Ren Fail. 2018;40:680-686. doi:10.1080/0886022X.2018.1544565

18. Wang BF, Cui ZW, Zhong ZH, et al. Curcumin attenuates brain edema in mice with intracerebral hemorrhage through inhibition of AQP4 and AQP9 expression. Acta Pharmacol Sin. 2015;36:939-948. doi:10.1038/aps.2015.47

19. de Souza Ferreira SB, Bruschi ML. Improving the bioavailability of curcumin: is micro/nanoencapsulation the key. Ther Deliv. 2019;10:83-86. doi:10.4155/tde-2018-0075

20. Yu Y, Zhang X, Qiu L. The anti-tumor efficacy of curcumin when delivered by size/charge-changing multistage polymeric micelles based on amphiphilic poly( $\beta$-amino ester) derivates. Biomaterials. 2014;35(10):3467-3479. doi:10.1016/j.biomaterials.2013.12.096

21. Li R, Deng L, Cai Z, et al. Liposomes coated with thiolated chitosan as drug carriers of curcumin. Mater Sci Eng C Mater Biol Appl. 2017;80:156-164. doi:10.1016/j.msec.2017.05.136

22. Luan L, Chi Z, Liu C. Chinese white wax solid lipid nanoparticles as a novel nanocarrier of curcumin for inhibiting the formation of staphylococcus aureus biofilms. Nanomaterials. 2019;9(5):763. doi:10.3390/nano9050763

23. Ten-Doménech I, Martínez-Pérez-Cejuela H, Simó-Alfonso EF, Torres-Cartas S, Meseguer-Lloret S, Herrero-Martínez JM. Polymerbased materials modified with magnetite nanoparticles for enrichment of phospholipids. Talanta. 2018;180:162-167. doi:10.1016/j. talanta.2017.12.042

24. Park K, Skidmore S, Hadar J, et al. Injectable, long-acting PLGA formulations: analyzing PLGA and understanding microparticle formation. J Control Release. 2019;304:125-134. doi:10.1016/j. jconrel.2019.05.003

25. Mondal L, Mukherjee B, Das K, et al. CD-340 functionalized doxorubicin-loaded nanoparticle induces apoptosis and reduces tumor volume along with drug-related cardiotoxicity in mice. Int J Nanomedicine. 2019;14:8073-8094. doi:10.2147/IJN.S220740

26. Dutta D, Paul B, Mukherjee B, et al. Nanoencapsulated betulinic acid analogue distinctively improves colorectal carcinoma in vitro and in vivo. Sci Rep. 2019;9(1):11506. doi:10.1038/s41598-019-47743-y

27. Asai M, Zhao D, Kumar SK. Accurate estimation of the polymer coverage of hairy nanoparticles. Soft Matter. 2018;14:7906-7915. doi:10.1039/C8SM01311J

28. Teresi R, Marullo S, Gambarotti C, et al. Improvement of oxidation resistance of polymer-based nanocomposites through sonication of carbonaceous nanoparticles. Ultrason Sonochem. 2020;61:104807. doi:10.1016/j.ultsonch.2019.104807

29. Hua Z, Jones JR, Thomas M, Arno MC, Souslov A, Wilks TR. Anisotropic polymer nanoparticles with controlled dimensions from the morphological transformation of isotropic seeds. Nat Commun. 2019;10(1):5406. doi:10.1038/s41467-019-13263-6

30. Cheng KK, Yeung CF, Ho SW, Chow SF, Chow AH, Baum L. Highly stabilized curcumin nanoparticles tested in an in vitro blood-brain barrier model and in Alzheimer's disease Tg2576 mice. AAPS J. 2013;15:324-336. doi:10.1208/s12248-012-9444-4

31. Wang Y, Song J, Chow SF, Chow AH, Zheng Y. Particle size tailoring of ursolic acid nanosuspensions for improved anticancer activity by controlled antisolvent precipitation. Int $J$ Pharm. 2015;494:479-489. doi:10.1016/j.ijpharm.2015.08.052

32. He B, Jia Z, Du W, et al. The transport pathways of polymer nanoparticles in MDCK epithelial cells. Biomaterials. 2013;34:4309-4326. doi:10.1016/j.biomaterials.2013.01.100

33. Fako VE, Furgeson DY. Zebrafish as a correlative and predictive model for assessing biomaterial nanotoxicity. Adv Drug Deliv Rev. 2009;61:478-486. doi:10.1016/j.addr.2009.03.008

34. Yu C, He B, Xiong MH, et al. The effect of hydrophilic and hydrophobic structure of amphiphilic polymeric micelles on their transport in epithelial MDCK cells. Biomaterials. 2013;34(26):6284-6298. doi:10.1016/j.biomaterials.2013.05.006 
35. Chen Z, Ma T, Huang C, et al. Efficiency of transcellular transport and efflux of flavonoids with different glycosidic units from flavonoids of Litsea coreana L. in a MDCK epithelial cell monolayer model. Eur J Pharm Sci. 2014;53:69-76. doi:10.1016/j.ejps.2013.12.010

36. Yang KY, Lin LC, Tseng TY, Wang SC, Tsai TH. Oral bioavailability of curcumin in rat and the herbal analysis from Curcuma longa by LC-MS/MS. J Chromatogr B Analyt Technol Biomed Life Sci. 2007;853(1-2):183-189. doi:10.1016/j.jchromb.2007.03.010

37. Mo Y, Duan L, Yang Y, et al. Nanoparticles improved resveratrol brain delivery and its therapeutic efficacy against intracerebral hemorrhage. Nanoscale. 2021;13:3827-3840. doi:10.1039/D0NR06249A

38. Sacks D, Baxter B, Campbell B, et al. Multisociety Consensus Quality Improvement Revised Consensus Statement for Endovascular Therapy of Acute Ischemic Stroke. Int J Stroke. 2018;13(6):612-632. doi:10.1177/1747493018778713

39. Lei W, Min W, Hui D, Yun L, An X. Effect of Surface Modification on Cellular Internalization of Fe3O4 Nanoparticles in Strong Static Magnetic Field. J Nanosci Nanotechnol. 2015;15(7):5184-5192. doi:10.1166/jnn.2015.9841

40. Jat D, Parihar P, Kothari SC, Parihar MS. Curcumin reduces oxidative damage by increasing reduced glutathione and preventing membrane permeability transition in isolated brain mitochondria. Cell $\mathrm{Mol}$ Biol. 2013;59(Supp1):OL1899-1905.

41. Marrache S, Dhar S. Engineering of blended nanoparticle platform for delivery of mitochondria-acting therapeutics. Proc Natl Acad Sci U S A. 2012;109(40):16288-16293. doi:10.1073/pnas.1210096109
42. Hauser AK, Mitov MI, Daley EF, McGarry RC, Anderson KW, Hilt JZ. Targeted iron oxide nanoparticles for the enhancement of radiation therapy. Biomaterials. 2016;105:127-135. doi:10.1016/j. biomaterials.2016.07.032

43. Wang HY, Cao PP, He ZY, et al. Targeted imaging and targeted therapy of breast cancer cells via fluorescent double template-imprinted polymer coated silicon nanoparticles by an epitope approach. Nanoscale. 2019;11(36):17018-17030. doi:10.1039/ C9NR04655K

44. Zhang Z, Wu Y, Yuan S, et al. Glutathione peroxidase 4 participates in secondary brain injury through mediating ferroptosis in a rat model of intracerebral hemorrhage. Brain Res. 2018;1701:112-125. doi:10.1016/j.brainres.2018.09.012

45. Imai H, Matsuoka M, Kumagai T, Sakamoto T, Koumura T. Lipid Peroxidation-Dependent Cell Death Regulated by GPx4 and Ferroptosis. Curr Top Microbiol Immunol. 2017;403:143-170. doi:10.1007/82_2016_508

46. Stockwell BR. A powerful cell-protection system prevents cell death by ferroptosis. Nature. 2019;575(7784):597-598. doi:10.1038/ d41586-019-03145-8

47. Yang Y, Luo M, Zhang K, et al. Nedd4 ubiquitylates VDAC2/3 to suppress erastin-induced ferroptosis in melanoma. Nat Commun. 2020;11(1):433. doi:10.1038/s41467-020-14324-x
International Journal of Nanomedicine

\section{Publish your work in this journal}

The International Journal of Nanomedicine is an international, peerreviewed journal focusing on the application of nanotechnology in diagnostics, therapeutics, and drug delivery systems throughout the biomedical field. This journal is indexed on PubMed Central, MedLine, CAS, SciSearch ${ }^{\mathbb{}}$, Current Contents ${ }^{\mathbb{R}} /$ Clinical Medicine,

\section{Dovepress}

Journal Citation Reports/Science Edition, EMBase, Scopus and the Elsevier Bibliographic databases. The manuscript management system is completely online and includes a very quick and fair peer-review system, which is all easy to use. Visit http://www.dovepress.com/ testimonials.php to read real quotes from published authors. 